\title{
SYSTEMS OF VARIATIONAL INEQUALITIES FOR NON-LOCAL OPERATORS RELATED TO OPTIMAL SWITCHING PROBLEMS: EXISTENCE AND UNIQUENESS
}

\author{
N. L. P. LUNDSTRÖM, K. NYSTRÖM, M. OLOFSSON
}

ABStRaCt. In this paper we study viscosity solutions to the system

$$
\begin{aligned}
& \min \left\{-\mathcal{H} u_{i}(x, t)-\psi_{i}(x, t), u_{i}(x, t)-\max _{j \neq i}\left(-c_{i, j}(x, t)+u_{j}(x, t)\right)\right\}=0, \\
& u_{i}(x, T)=g_{i}(x), i \in\{1, \ldots, d\},
\end{aligned}
$$

where $(x, t) \in \mathbb{R}^{N} \times[0, T]$. Concerning $\mathcal{H}$ we assume that $\mathcal{H}=\mathcal{L}+\mathcal{I}$ where $\mathcal{L}$ is a linear, possibly degenerate, parabolic operator of second order and $\mathcal{I}$ is a non-local integro-partial differential operator. A special case of this type of system of variational inequalities with terminal data occurs in the context of optimal switching problems when the dynamics of the underlying state variables is described by an $N$-dimensional Levy process. We establish a general comparison principle for viscosity sub- and supersolutions to the system under mild regularity, growth and structural assumptions on the data, i.e., on the operator $\mathcal{H}$ and on continuous functions $\psi_{i}, c_{i, j}$, and $g_{i}$. Using the comparison principle we prove the existence of a unique viscosity solution $\left(u_{1}, \ldots, u_{d}\right)$ to the system by Perron's method. Our main contribution is that we establish existence and uniqueness of viscosity solutions, in the setting of Levy processes and non-local operators, with no sign assumption on the switching costs $\left\{c_{i, j}\right\}$ and allowing $c_{i, j}$ to depend on $x$ as well as $t$. 2000 Mathematics Subject Classification.

Keywords and phrases: system, variational inequality, existence, viscosity solution, non-local operator, integro-partial differential operator, Levy process, jump-diffusion, optimal switching problem.

N.L.P. Lundström and M. Olofsson were financed by Jan Wallanders och Tom Hedelius Stiftelse samt Tore Browaldhs Stiftelse through the project Optimal switching problems and their applications in economics and finance, P2010-0033:1. 


\section{IntRoduCtion AND STATEMENT OF MAIN RESUlts}

In this paper we consider the problem

$$
\begin{aligned}
& \min \left\{-\mathcal{H} u_{i}(x, t)-\psi_{i}(x, t), u_{i}(x, t)-\max _{j \neq i}\left(-c_{i, j}(x, t)+u_{j}(x, t)\right)\right\}=0, \\
& u_{i}(x, T)=g_{i}(x), i \in\{1, \ldots, d\}
\end{aligned}
$$

in $\mathbb{R}^{N} \times[0, T]\left((x, t) \in \mathbb{R}^{N} \times[0, T]\right), T>0$, where $\psi_{i}, c_{i, j}$, and $g_{i}$ are continuous functions and $\mathcal{H}$ is a non-local integro-partial differential operator. Concerning $\mathcal{H}$ we assume that $\mathcal{H}=\mathcal{L}+\mathcal{I}$,

$$
\mathcal{L}=\sum_{i, j=1}^{N} a_{i, j}(x, t) \partial_{x_{i} x_{j}}+\sum_{i=1}^{N} a_{i}(x, t) \partial_{x_{i}}+\partial_{t},
$$

for continuous functions $a_{i, j}$ and $a_{i}$, and that $\mathcal{I}$ is a non-local integro-partial differential operator which for smooth functions $\phi$ is defined as

$$
\begin{aligned}
\mathcal{I}(x, t, \phi)= & \int_{\mathbb{R}^{l} \backslash\{0\}} K(x, t, z, \phi, D \phi) \nu(d z), \\
K(x, t, z, \phi, p)= & \phi(x+\eta(x, t, z), t)-\phi(x, t) \\
& -\chi_{\{|z| \leq 1\}}(z) \sum_{k=1}^{N} \eta_{k}(x, t, z) p_{k},
\end{aligned}
$$

where $(x, t) \in \mathbb{R}^{N} \times \mathbb{R}, D \phi=\left(\partial_{x_{1}} \phi, \ldots, \partial_{x_{N}} \phi\right), p \in \mathbb{R}^{N}$. Here $\nu$ is a positive Radon measure and, for each $i \in\{1, \ldots, N\}, \eta_{i}$ is a function taking values in $\mathbb{R}^{N} \cdot \chi_{\{|z| \leq 1\}}$ is the indicator function for the closed unit ball in $\mathbb{R}^{l}$. Operators $\mathcal{H}=\mathcal{L}+\mathcal{I}$ occur, for instance, in the context of financial markets where the dynamics of the state variables is described by an $N$-dimensional Levy process $X=\left(X_{s}^{x, t}\right)$ solving the system of stochastic differential equations

$$
\begin{aligned}
d X_{s}^{x, t}= & a\left(X_{s}^{x, t}, s\right) d s+\sigma\left(X_{s}^{x, t}, s\right) d W_{s} \\
& +\int_{z \in \mathbb{R}^{l}} \eta\left(X_{s^{-}}^{x, t}, s, z\right) d \tilde{N}(d s, d z),
\end{aligned}
$$

for $t \leq s \leq T$, with initial condition $X_{s}^{x, t}=x, 0 \leq s \leq t,(x, t) \in \mathbb{R}^{N} \times[0, T]$. Here $W=\left\{W_{t}\right\}$ denotes a standard $N$-dimensional Brownian motion and

$$
\tilde{N}(d s, d z)= \begin{cases}\hat{N}(d s, d z) & \text { if }|z| \geq 1 \\ \hat{N}(d s, d z)-\nu(d z) d s & \text { if }|z|<1\end{cases}
$$

where $\hat{N}$ is a Poisson random measure on $[0, \infty) \times \mathbb{R}^{l}$ with intensity measure $\nu(d z) d s$. $\mathcal{H}$ can, in the context of (1.4), be seen as the infinitesimal generator associated to $X=\left(X_{s}^{x, t}\right)$ and we note that the diffusion part of the system then is described by the $N \times N$-dimensional matrix $\sigma$. The process $X=\left(X_{s}^{x, t}\right)$ can, for instance, be the electricity price or other factors which determine the price. In the Markovian setting when the randomness stems from the Levy process $X=\left(X_{s}^{x, t}\right)$ in (1.4), the problem in (1.1) is a system of variational inequalities with inter-connected obstacles related to multi-modes optimal switching problems. Our main results concern existence and uniqueness of viscosity solutions to the system in (1.1) under mild regularity, growth and structural assumptions on the data, i.e., on the operator $\mathcal{H}$ and on continuous functions $\psi_{i}, c_{i, j}$, and $g_{i}$.

In multi-modes optimal switching problems the system in (1.1) occurs with $g=$ $\left(g_{1}, \ldots, g_{d}\right) \equiv(0, \ldots, 0)$. To outline the setting for this class of problems, consider a production facility which can run the production in $d, d \geq 2$, production modes. Let $X=\left(X_{s}^{x, t}\right)$ denote the vector of stochastic processes in (1.4) which, as discussed 
above, represents the market price of the underlying commodities and other finance assets that influence the production. Let the payoff rate in production mode $i$, at time $t$, be $\psi_{i}\left(X_{t}, t\right)$ and let $c_{i, j}\left(X_{t}, t\right)$ denote the switching cost for switching from mode $i$ to mode $j$ at time $t$. A management strategy is a combination of a nondecreasing sequence of stopping times $\left\{\tau_{k}\right\}_{k>0}$, where, at time $\tau_{k}$, the manager decides to switch production from its current mode to another one, and a sequence of indicators $\left\{\xi_{k}\right\}_{k \geq 0}$, taking values in $\{1, \ldots, d\}$, indicating the mode to which the production is switched. At $\tau_{k}$ the production is switched from mode $\xi_{k-1}$ (current mode) to $\xi_{k}$. A strategy $\left(\left\{\tau_{k}\right\}_{k \geq 0},\left\{\xi_{k}\right\}_{k \geq 0}\right)$ can be represented by the simple function

$$
\mu_{s}=\sum_{i \geq 1} \xi_{i} \chi_{\left(\tau_{i}, \tau_{i+1}\right]}(s)+\xi_{0} \chi_{\left[\tau_{0}, \tau_{1}\right]}(s) .
$$

When the production is run using a strategy $\mu$, defined by $\left(\left\{\tau_{k}\right\}_{k \geq 0},\left\{\xi_{k}\right\}_{k \geq 0}\right)$, over a finite horizon $[0, T]$, the total expected profit up to time $T$ is

$$
J(\mu)=E\left[\left(\int_{0}^{T} \psi_{\mu_{s}}\left(X_{s}, s\right) d s-\sum_{k \geq 1} c_{\xi_{k-1}, \xi_{k}}\left(X_{\tau_{k}}, \tau_{k}\right)\right)\right] .
$$

The optimal switching problem consists of finding an optimal management strategy $\mu^{*}$, defined by $\left(\left\{\tau_{k}^{*}\right\}_{k \geq 0},\left\{\xi_{k}^{*}\right\}_{k \geq 0}\right)$, such that

$$
J\left(\mu^{*}\right)=\sup _{\mu} J(\mu) .
$$

Let $\left(Y_{t}^{1}, \ldots, Y_{t}^{d}\right)$ be the value function associated with the optimal switching problem, on the time interval $[t, T]$, where $Y_{t}^{i}$ stands for the optimal expected profit if, at time $t$, the production is in mode $i$. Under sufficient assumptions, see for example BJK10, it can then be proved that $\left(Y_{t}^{1}, \ldots, Y_{t}^{d}\right)=\left(u_{1}\left(X_{t}, t\right), \ldots, u_{d}\left(X_{t}, t\right)\right)$, where the vector of deterministic functions $\left(u_{1}(x, t), \ldots, u_{d}(x, t)\right)$ satisfies (1.1) with $g=\left(g_{1}, \ldots, g_{d}\right) \equiv(0, \ldots, 0)$.

1.1. Assumptions. We here outline the assumptions we impose on $\mathcal{H}$ and on the functions $\psi_{i}, c_{i, j}$, and $g_{i}$. Firstly, focusing on $\mathcal{H}=\mathcal{L}+\mathcal{I}$ we impose additional structural assumptions on the matrix $\left\{a_{i, j}\right\}_{i, j=1}^{N}$. In particular, we assume that

$$
a_{i, j}(x, t)=\left(\sigma(x, t) \sigma^{*}(x, t)\right)_{i, j}, i, j \in\{1, \ldots, N\},
$$

where $\sigma=\sigma(x, t)$ is an $N \times N$ matrix and $\sigma^{*}$ is the transpose of $\sigma$. Concerning regularity and growth conditions on $\left\{a_{i, j}\right\}_{i, j=1}^{N}$ and $\left\{a_{i}\right\}_{i=1}^{N}$, we assume that

$$
\begin{aligned}
\text { (i) } & \left|a_{i}(x, t)-a_{i}(y, s)\right|+\left|\sigma_{i, j}(x, t)-\sigma_{i, j}(y, s)\right| \leq A|x-y|, \\
(i i) & \left|a_{i}(x, t)\right|+\left|\sigma_{i, j}(x, t)\right| \leq A(1+|x|),
\end{aligned}
$$

for some $A, 1 \leq A<\infty$, for all $i, j \in\{1, \ldots, N\}$, and whenever $(x, t),(y, s) \in$ $\mathbb{R}^{N} \times[0, T]$. Here, $|x|$ is the standard Euclidean norm of $x \in \mathbb{R}^{N}$. Note that $(i)$ implies (ii) in (1.9). Note also that $\left\{a_{i, j}\right\}_{i, j=1}^{N}$ is only assumed non-negative definite and hence large sets of entries in the matrices $\left\{a_{i, j}\right\}_{i, j=1}^{N},\left\{\sigma_{i, j}\right\}_{i, j=1}^{N}$, may be zero at points resulting in degeneracies.

The above restrictions define the local part, $\mathcal{L}$, of $\mathcal{H}$. Focusing on the non-local part, $\mathcal{I}$, of $\mathcal{H}$, we assume that $\nu$ is a positive Radon measure defined on $\mathbb{R}^{l} \backslash\{0\}$ such that

$$
\int_{0<|z| \leq 1}|z|^{2} \nu(d z)+\int_{1<|z|} e^{\Lambda|z|} \nu(d z) \leq \tilde{A}
$$

for some constant $\tilde{A}, 1 \leq \tilde{A}<\infty$, and for some $\Lambda>0$. In addition we assume that $\eta_{k}$ is, for $k \in\{1, \ldots, N\}$, continuous in $x$ and $t$, Borel measurable in $z$, and that

$$
\left|\eta_{k}(x, t, z)\right| \leq \tilde{B} \min \{|z|, 1\}
$$




$$
\left|\eta_{k}(x, t, z)-\eta_{k}(y, t, z)\right| \leq \tilde{B} \min \{|z|, 1\}|x-y|,
$$

for some constant $\tilde{B}, 1 \leq \tilde{B}<\infty$, and for all $x, y \in \mathbb{R}^{N}, t \in \mathbb{R}, z \in \mathbb{R}^{l}$. This completes our definition of $\mathcal{H}$. Secondly, concerning regularity and growth conditions on $\psi_{i}, c_{i, j}$ and $g_{i}$ we assume that

$$
\begin{aligned}
& \psi_{i}, c_{i, j} \text { and } g_{i} \text { are continuous functions, } \\
& \left|\psi_{i}(x, t)\right|+\left|c_{i, j}(x, t)\right|+\left|g_{i}(x)\right| \leq B\left(1+|x|^{\gamma}\right) \text { for some } \\
& B, \gamma \in[1, \infty), \text { whenever }(x, t) \in \mathbb{R}^{N} \times[0, T] .
\end{aligned}
$$

Thirdly, the structural assumptions on the functions $\left\{c_{i, j}\right\}$ that we impose to establish our general comparison principle for the system in (1.1), see Theorem 1.1. are the following.

$$
c_{i, i}(x, t)=0 \text { for each } i \in\{1, \ldots, d\},
$$

(ii) For any sequence $i_{1}, \ldots, i_{k}, i_{j} \in\{1, \ldots, d\}$ for each $j \in\{1, \ldots, k\}$,

$$
\begin{aligned}
& \text { we have } c_{i_{1}, i_{2}}(x, t)+c_{i_{2}, i_{3}}(x, t)+\cdots+c_{i_{k-1}, i_{k}}(x, t)+c_{i_{k}, i_{1}}(x, t)>0, \\
& \text { for all }(x, t) \in \mathbb{R}^{N} \times[0, T] .
\end{aligned}
$$

Finally, concerning the interplay between the terminal data $\left\{g_{i}\right\}$ and the switching $\operatorname{costs}\left\{c_{i, j}\right\}$, at $t=T$, we assume that

$$
g_{i}(x) \geq \max _{j \neq i}\left(-c_{i, j}(x, T)+g_{j}(x)\right)
$$

for all $i \in\{1, \ldots, d\}$, and for all $x \in \mathbb{R}^{N}$. Note that in the special case of the optimal switching problem discussed above, i.e., $g=\left(g_{1}, \ldots, g_{d}\right) \equiv(0, \ldots, 0)$, then (1.14) implies that $c_{i, j}(x, T) \geq 0$ for all $i, j \in\{1, \ldots, d\}$, and for all $x \in \mathbb{R}^{N}$. Focusing on the structural assumptions on the functions $\left\{c_{i, j}\right\}$, we emphasize that in our final existence theorem, see Theorem 1.3 we assume regularity of $c_{i, j}(x, t)$ beyond continuity, see (1.16), and that

$$
c_{i_{1}, i_{2}}(x, t)+c_{i_{2}, i_{3}}(x, t) \geq c_{i_{1}, i_{3}}(x, t)
$$

for any sequence of indices $i_{1}, i_{2}, i_{3}, i_{l} \in\{1, \ldots, d\}$ for each $l \in\{1,2,3\}$, and for all $(x, t) \in \mathbb{R}^{N} \times[0, T]$. Note that (1.15) is an additional structural restriction compared to (1.13).

1.2. Statement of main results. We here formulate our main results. For the definition of $\operatorname{LSC}_{p}\left(\mathbb{R}^{N} \times[0, T]\right), \mathrm{USC}_{p}\left(\mathbb{R}^{N} \times[0, T]\right)$ and $\mathrm{C}_{p}\left(\mathbb{R}^{N} \times[0, T]\right)$ as well as for the definition of viscosity sub- and supersolutions we refer to the bulk of the paper. We first prove the following comparison principle.

Theorem 1.1. Let $\mathcal{H}=\mathcal{L}+\mathcal{I}$ with $\mathcal{L}$, $\mathcal{I}$, as in (1.2), (1.3), respectively. Assume (1.8), (1.9), (1.10), (1.11), (1.12), (1.13) and (1.14). Assume that $\left(u_{1}^{+}, \ldots, u_{d}^{+}\right) \in$ $L S C_{p}\left(\mathbb{R}^{N} \times[0, T]\right)$ and $\left(u_{1}^{-}, \ldots, u_{d}^{-}\right) \in U S C_{p}\left(\mathbb{R}^{N} \times[0, T]\right)$ are viscosity super- and subsolutions, respectively, to the problem in (1.1). Then $u_{i}^{-} \leq u_{i}^{+}$in $\mathbb{R}^{N} \times(0, T]$ for all $i \in\{1, \ldots, d\}$.

Before stating our existence theorems, we make the following definition.

Definition 1. A barrier from above for the system in (1.1), component $i \in$ $\{1, \ldots, d\}$ and the point $y \in \mathbb{R}^{N}, u^{+, i, y}$, is a family of continuous supersolutions, $\left\{u^{+, i, y, \varepsilon}\right\}_{\varepsilon>0}, u^{+, i, y, \varepsilon} \in \mathrm{C}_{p}\left(\mathbb{R}^{N} \times[0, T]\right)$, to system (1.1) such that

$$
\lim _{\varepsilon \rightarrow 0} u_{i}^{+, i, y, \varepsilon}(y, T)=g_{i}(y) .
$$

A barrier from below for the system in 11.1), component $i \in\{1, \ldots, d\}$ and the point $y \in \mathbb{R}^{N}, u^{-, i, y}$, is a family of continuous subsolutions, $\left\{u^{-, i, y, \varepsilon}\right\}_{\varepsilon>0}, u^{-, i, y, \varepsilon} \in$ 
$\mathrm{C}_{p}\left(\mathbb{R}^{N} \times[0, T]\right)$, to system (1.1) such that

$$
\lim _{\varepsilon \rightarrow 0} u_{i}^{-, i, y, \varepsilon}(y, T)=g_{i}(y) \text {. }
$$

To stress generality, we first prove the following theorem.

Theorem 1.2. Let $\mathcal{H}=\mathcal{L}+\mathcal{I}$ with $\mathcal{L}, \mathcal{I}$, as in (1.2), (1.3), respectively. Assume (1.8), (1.9), (1.10), (1.11), (1.12), (1.13) and (1.14). In addition, assume that

(1) there exists, for each $i \in\{1, \ldots, d\}$ and $y \in \mathbb{R}^{N}$, a barrier from above $u^{+}=u^{+, i, y}$ to the system in (1.1) in the sense of Definition 1 ,

(2) there exists, for each $i \in\{1, \ldots, d\}$ and $y \in \mathbb{R}^{N}$, a barrier from below $u^{-}=u^{-, i, y}$ to the system in (1.1) in the sense of Definition 1 .

Then there exists a viscosity solution $\left(u_{1}, \ldots, u_{d}\right) \in C_{p}\left(\mathbb{R}^{N} \times[0, T]\right)$ to the problem in (1.1), $u_{i}^{-} \leq u_{i} \leq u_{i}^{+}$on $\mathbb{R}^{N} \times[0, T]$, for $i \in\{1, \ldots, d\}$, and this solution is unique in the class $C_{p}\left(\mathbb{R}^{N} \times[0, T]\right)$.

To establish existence of a viscosity solution $\left(u_{1}, \ldots, u_{d}\right)$ to the problem in (1.1), it hence remains to construct barriers in the sense of Definition 11. To do so we impose additional assumptions on the switching $\operatorname{costs} c_{i, j}$, see Theorem 1.3 below. In particular, we prove that for fixed $i \in\{1, \ldots, d\}, y \in \mathbb{R}^{N}$ and all $j \in\{1, \ldots, d\}$,

$$
\begin{aligned}
u_{j}^{+, i, y, \varepsilon}(x, t)= & g(y)+\frac{K}{\varepsilon^{2}}(T-t) \\
& +L\left(e^{\lambda(T-t)}+1\right)\left(|x-y|^{2}+\varepsilon\right)^{\frac{1}{2}}+c_{i, j}(x, t),
\end{aligned}
$$

is a barrier from above if $K$ and $\lambda$ are large enough. Here $L$ is the Lipschitz-constant of $g(x)$. Since $c_{i, i}=0$ by assumption, $u_{j}^{+, i, y, \varepsilon}$ attains the terminal data $g$ as $\varepsilon \rightarrow 0$ and hence, from Theorem 1.2 we now deduce existence of a viscosity solution to the problem in (1.1). In particular, we prove the following theorem.

Theorem 1.3. Let $\mathcal{H}=\mathcal{L}+\mathcal{I}$ with $\mathcal{L}, \mathcal{I}$, as in (1.2), (1.3), respectively. Assume (1.8), (1.9), (1.10), (1.11), (1.12), (1.13) and (1.14). Assume also that $g_{i}=g$, for all $i \in\{1, \ldots, d\}$, for some Lipschitz continuous function $g$, that $c_{i, j} \in C^{1,2}\left(\mathbb{R}^{N} \times\right.$ $[0, T))$, and that (1.15) holds for any sequence of indices $i_{1}, i_{2}, i_{3}, i_{j} \in\{1, \ldots, d\}$ for each $j \in\{1,2,3\}$, and for all $(x, t) \in \mathbb{R}^{N} \times[0, T]$. Furthermore, assume that

$$
\begin{aligned}
& \partial_{x_{k}} c_{i, j} \in L^{\infty}\left(\mathbb{R}^{N} \times[0, T]\right) \\
& \left|\psi_{i}(x, t)\right|,|g(x, t)|,\left|\partial_{t} c_{i, j}(x, t)\right|,\left|\partial_{x_{k} x_{l}} c_{i, j}(x, t)\right|\left(1+|x|^{2}\right) \leq \tilde{A}(1+|x|)
\end{aligned}
$$

for all $(x, t) \in \mathbb{R}^{N} \times[0, T]$ and for some $\tilde{A}, 1 \leq \tilde{A}<\infty$. Then there exists a unique viscosity solution $\left(u_{1}, \ldots, u_{d}\right)$ to the problem in (1.1), unique in the sense defined in Theorem 1.2.

Remark 1.1. Through $\mathcal{H}=\mathcal{L}+\mathcal{I}$ we see that $\mathcal{H}$ is written as the sum of the local operator $\mathcal{L}$ and the non-local operator $\mathcal{I}$. Note that by the assumptions in Theorem 1.1.Theorem 1.3 the matrix $\left\{a_{i, j}(x, t)\right\}$ is only assumed to be non-negative definite and as such it can vanish at points. Similarly the jump vector $\eta=\eta(x, t, z)$ is allowed to vanish. A consequence of this is that there are no regularization effects in the problem coming either from $\mathcal{L}$ or $\mathcal{I}$. Therefore the system in (1.1) can not be expected to have classical solutions and hence an appropriate notion of viscosity solutions is needed.

Remark 1.2. $\mathcal{H}$ can be seen as the infinitesimal generator associated with a Levy process described by (1.4). In this context, the assumptions stated in (1.10) exclude some Levy processes as a Levy measure $\nu$ in general only satisfies

$$
\int \min \left\{|z|^{2}, 1\right\} \nu(d z)<\infty .
$$


However, for many, if not most, applications the class of Levy processes considered in this paper is sufficiently rich since, e.g., any Levy process with compactly supported Levy measure satisfies (1.10).

Remark 1.3. In BJK10 the authors basically prove Theorem 1.1 Theorem 1.3 for a combined optimal switching and control problem essentially assuming $\mathcal{H}=\mathcal{L}+\mathcal{I}$ with $\mathcal{L}, \mathcal{I}$, as in (1.2), (1.3), respectively, and (1.8), (1.9), (1.10), (1.11), and (1.12) with $\gamma=2$. Our contribution is that we prove Theorem 1.1 Theorem 1.3 allowing for much more general switching costs compared to BJK10.

Remark 1.4. Our proofs of Theorem 1.1 Theorem 1.3 are influenced by the corresponding arguments in BJK10, AF12, and by the arguments in LNO12 where versions of 1.1 Theorem 1.3 are proved in the case when $\mathcal{H} \equiv \mathcal{L}$, i.e., in the case of local operators. In the latter paper the problem of regularity of viscosity solutions was also treated in the context of operators of Kolmogorov type.

Remark 1.5. Theorem 1.17Theorem 1.3 remain true for the more general nonlinear problems

$$
\begin{aligned}
& \min \left\{-\sup _{\alpha \in A_{i}} \inf _{\beta \in B_{i}}\left[\mathcal{H}_{\alpha, \beta}^{i} u_{i}(x, t)-\psi_{i}^{\alpha, \beta}(x, t)\right],\right. \\
& \left.u_{i}(x, t)-\max _{j \neq i}\left(-c_{i, j}(x, t)+u_{j}(x, t)\right)\right\}=0, \\
& u_{i}(x, T)=g_{i}(x), i \in\{1, \ldots, d\},
\end{aligned}
$$

where $A_{i}, B_{i}$ are compact metric spaces and $\mathcal{H}_{\alpha, \beta}^{i}=\mathcal{L}_{\alpha, \beta}^{i}+\mathcal{I}_{\alpha, \beta}^{i}$, for each $i \in$ $\{1, \ldots, d\}$, is as in (1.2), (1.3), for some $\sigma_{k, l, \alpha, \beta}^{i}, a_{k, l, \alpha, \beta}^{i}, a_{k, \alpha, \beta}^{i}, \nu, \eta_{\alpha, \beta}^{i}$ satisfying (1.8), (1.9), (1.10), and (1.11) uniformly in $\alpha, \beta$. Equations of this type are considered in [BJK10] and arise for example as the Bellman-Isaacs equations for zero-sum stochastic games.

1.3. Our contribution in relation to the current literature. In this paper we consider optimal switching problems for operators $\mathcal{H}=\mathcal{L}+\mathcal{I}$, where $\mathcal{L}$ is a local operator and $\mathcal{I}$ is non-local operator, allowing for general switching costs. As a general comment we note that there is currently a substantial literature devoted to the the case of local operators, i.e., $\mathcal{H} \equiv \mathcal{L}$, and that considerably less is known in the case when non-local effects are allowed.

In the local setting there is a well-established connection between the theory of reflected backward stochastic differential equations driven by Brownian motion and multi-modes optimal switching problems, see AF12, DH09, DHP10, HT07] and HZ10. In general the research on optimal switching problems focuses on two approaches: the probabilistic or stochastic approach and the deterministic approach. We note that the stochastic approach heavily uses probabilistic tools such as the Snell envelope and backward stochastic differential equations. For more on the stochastic approach we refer to the references above concerning reflected backward stochastic differential equations. The latter, deterministic approach focuses more on variational inequalities and partial differential equations and we refer, in the local setting, to AH09 and LNO12. In general, in the local setting the two approaches are used in combination due to the connection between reflected backward stochastic differential equations and systems of variational inequalities. The results in the literature concerning the optimal switching problem, i.e., system (1.1) with $g=\left(g_{1}, \ldots, g_{d}\right) \equiv(0, \ldots, 0)$, are derived under different assumptions on the switching costs $\left\{c_{i, j}\right\}$. We note that the switching costs in the references listed above are essentially always assumed to be non-negative, i.e., $c_{i, j}(x, t) \geq \alpha$ for all $(x, t) \in \mathbb{R}^{N} \times[0, T], i, j \in\{1, \ldots, d\}, i \neq j$, and for some $\alpha \geq 0$. Often, even $\alpha>0$ 
is assumed, see [AH09] and DH09 for instance. Furthermore, often additional restrictions are imposed on $\left\{c_{i, j}\right\}$, like $c_{i, j}$ is independent of $x$, see [DHP10] for instance, or that $c_{i, j}$ is even constant, see DH09, BJK10. The only papers we are aware of where the switching costs are allowed to change sign are AF12 and LNO12. However, in AF12 there are two additional conditions concerning the non-negativity of the switching costs: $c_{i, j}(x, T)=0$ and the number of negative switching costs is limited in a certain sense, see condition $(v)$ in [AF12. In LNO12] the assumptions imposed on the switching costs are in line with the assumptions imposed in this paper.

In the non-local setting the connections between a theory of reflected backward stochastic differential equation driven by Levy processes and multi-modes optimal switching problem seems to be considerably less developed. In general the theory for non-local operators is currently an active area of research. We here refer to A07, BI08, BJK10 and P97. for an account of this and for references, but to our knowledge BJK10] is the only paper that makes contribution to multi-mode optimal switching problems involving non-local operators, i.e., involving non-local effects through Levy processes that are allowed to jump. Building on BJK10 our main contribution is that while in BJK10] the switching costs are assumed to be non-negative constants, our baseline assumption on the functions $\left\{c_{i, j}\right\}$ is that we make no sign assumption on the switching costs $\left\{c_{i, j}\right\}$ and that $c_{i, j}$ is allowed to depend on $x$ as well as $t$. Naturally one may ask whether the allowance for possibly negative switching costs is of importance beyond the mathematical issues. In fact, it is very natural to allow for negative switching costs as these allow one to model the situation when, for example, a government through environmental policies provide subsidies, grants or other financial support to energy production facilities in case they switch to more 'green energy' production or to more clean methods for production. In this case it is not a cost for the facility to switch, it is a gain. However, the final decision to switch or not to switch is naturally also influenced by the running pay-offs $\left\{\psi_{i}\right\}$.

1.4. Outline of the paper. The rest of this paper is organized as follows. Section 2 is of preliminary nature and we here introduce some notation and state the definition of viscosity subsolutions, supersolutions and solutions. Section 3 is devoted to the proof of Theorem 1.1 and in section 4 we prove Theorem 1.2 and Theorem 1.3

\section{Preliminaries}

In this section we introduce some notation used throughout the paper and we define viscosity sub- and supersolutions to the problem in (1.1).

2.1. Notation. We denote by $\operatorname{LSC}\left(\mathbb{R}^{N} \times[0, T]\right)$ the set of lower semi-continuous functions, i.e., all functions $f: \mathbb{R}^{N} \times[0, T] \rightarrow \mathbb{R}$ such that for all points $\left(x_{0}, t_{0}\right)$ and for any sequence $\left\{\left(x_{n}, t_{n}\right)\right\}_{n}, \lim _{n \rightarrow \infty}\left(x_{n}, t_{n}\right) \rightarrow\left(x_{0}, t_{0}\right)$ in $\mathbb{R}^{N} \times[0, T]$, we have

$$
\liminf _{n \rightarrow \infty} f\left(x_{n}, t_{n}\right) \geq f\left(x_{0}, t_{0}\right) .
$$

Likewise, we denote by $\operatorname{USC}\left(\mathbb{R}^{N} \times[0, T]\right)$ the set of upper semi-continuous functions, i.e., all functions $f: \mathbb{R}^{N} \times[0, T] \rightarrow \mathbb{R}$ such that for all points $\left(x_{0}, t_{0}\right)$ and for any sequence $\left\{\left(x_{n}, t_{n}\right)\right\}_{n}, \lim _{n \rightarrow \infty}\left(x_{n}, t_{n}\right) \rightarrow\left(x_{0}, t_{0}\right)$ in $\mathbb{R}^{N} \times[0, T]$, we have

$$
\limsup _{n \rightarrow \infty} f\left(x_{n}, t_{n}\right) \leq f\left(x_{0}, t_{0}\right) \text {. }
$$

Note that a function $f$ is upper semi-continuous if and only if $-f$ is lower semicontinuous. Also, a real function $g$ is continuous if and only if it is both upper and lower semi-continuous. Given $\gamma \in[1, \infty)$, the function space $\operatorname{LSC}_{p, \gamma}\left(\mathbb{R}^{N} \times[0, T]\right)$ 
is defined to consist of functions $h \in \operatorname{LSC}\left(\mathbb{R}^{N} \times[0, T]\right)$ which satisfy the growth condition

$$
|h(x, t)| \leq c\left(1+|x|^{\gamma}\right)
$$

for some $c \in[1, \infty)$, whenever $(x, t) \in \mathbb{R}^{N} \times[0, T]$. USC $\mathrm{US}_{p, \gamma}\left(\mathbb{R}^{N} \times[0, T]\right)$ is defined by analogy. Furthermore, $\mathrm{C}_{p, \gamma}\left(\mathbb{R}^{N} \times[0, T]\right)=\mathrm{USC}_{p, \gamma}\left(\mathbb{R}^{N} \times[0, T]\right) \cap \mathrm{LSC}_{p, \gamma}\left(\mathbb{R}^{N} \times\right.$ $[0, T]) . \quad \mathrm{C}_{p}\left(\mathbb{R}^{N} \times[0, T]\right), \mathrm{USC}_{p}\left(\mathbb{R}^{N} \times[0, T]\right), \mathrm{LSC}_{p}\left(\mathbb{R}^{N} \times[0, T]\right)$ are the unions, respectively, of the sets $\mathrm{C}_{p, \gamma}\left(\mathbb{R}^{N} \times[0, T]\right), \mathrm{USC}_{p, \gamma}\left(\mathbb{R}^{N} \times[0, T]\right), \mathrm{LSC}_{p, \gamma}\left(\mathbb{R}^{N} \times[0, T]\right)$, with respect to $\gamma \geq 1$. By $C^{1,2}\left(\mathbb{R}^{N} \times[0, T)\right)$ we denote the set of functions which are twice continuously differentiable in the spatial variables and once continuously differentiable in the time variable, on $\mathbb{R}^{N} \times[0, T)$. We will by $c$ denote a generic constant, $1 \leq c<\infty$, that may change value from line to line.

2.2. Viscosity solutions. We here define the notion of viscosity solutions to the problem in (1.1). Let $\mathcal{H}=\mathcal{L}+\mathcal{I}$ with $\mathcal{L}, \mathcal{I}$, as in (1.2), (1.3), respectively. Given $(x, t) \in \mathbb{R}^{N} \times[0, T], \phi \in C^{1,2}\left(\mathbb{R}^{N} \times[0, T)\right), p \in \mathbb{R}^{N}, \kappa \in(0,1)$, we let

$$
\begin{aligned}
\mathcal{I}_{\kappa}(x, t, \phi, p) & =\int_{\{z:|z|<\kappa\}} K(x, t, z, \phi, p) \nu(d z), \\
\mathcal{I}^{\kappa}(x, t, \phi, p) & =\int_{\{z:|z| \geq \kappa\}} K(x, t, z, \phi, p) \nu(d z),
\end{aligned}
$$

where

$$
\begin{aligned}
K(x, t, z, \phi, p)= & \phi(x+\eta(x, t, z), t)-\phi(x, t) \\
& -\chi_{\{|z| \leq 1\}}(z) \sum_{k=1}^{N} \eta_{k}(x, t, z) p_{k} .
\end{aligned}
$$

Note that $\mathcal{I}=\mathcal{I}_{\kappa}+\mathcal{I}^{\kappa}$ and that $\mathcal{I}_{\kappa}$ and $\mathcal{I}^{\kappa}$ give, respectively, the contribution to $\mathcal{I}$ from the 'small' and 'large' jumps. Using this notation we let $\mathcal{H}^{\kappa}(\phi, u)=$ $\mathcal{H}^{\kappa}(x, t, \phi, u)$ where

$$
\mathcal{H}^{\kappa}(x, t, \phi, u):=\mathcal{L} \phi(x, t)+\mathcal{I}_{\kappa}(x, t, \phi, D \phi)+\mathcal{I}^{\kappa}(x, t, u, D \phi)
$$

whenever $(x, t) \in \mathbb{R}^{N} \times[0, T], \phi \in C^{1,2}\left(\mathbb{R}^{N} \times[0, T)\right)$ and $u \in \operatorname{LSC}_{p}\left(\mathbb{R}^{N} \times[0, T]\right) \cup$ $\mathrm{USC}_{p}\left(\mathbb{R}^{N} \times[0, T]\right)$.

Definition 2. (i) A vector $\left(u_{1}^{+}, \ldots, u_{d}^{+}\right), u_{i} \in \operatorname{LSC}_{p}\left(\mathbb{R}^{N} \times[0, T]\right)$ for $i \in\{1, \ldots, d\}$, is a viscosity supersolution to the problem in (1.1) if $u_{i}^{+}(x, T) \geq g_{i}(x)$ whenever $x \in \mathbb{R}^{N}, i \in\{1, \ldots, d\}$, and if the following holds. If $\left(x_{0}, t_{0}\right) \in \mathbb{R}^{N} \times(0, T)$ and if, for some $i \in\{1, \ldots, d\}$, we have $\phi_{i} \in C^{1,2}\left(\mathbb{R}^{N} \times[0, T)\right)$ such that

(i) $\phi_{i}\left(x_{0}, t_{0}\right)=u_{i}^{+}\left(x_{0}, t_{0}\right)$

(ii) $\quad\left(x_{0}, t_{0}\right)$ is a global maximum of $\phi_{i}-u_{i}^{+}$,

then

$$
\min \left\{-\mathcal{H}^{\kappa}\left(\phi_{i}, u_{i}^{+}\right)-\psi_{i}, u_{i}^{+}-\max _{j \neq i}\left(-c_{i, j}+u_{j}^{+}\right)\right\} \geq 0 \text { for all } \kappa \in(0,1) .
$$

(ii) A vector $\left(u_{1}^{-}, \ldots, u_{d}^{-}\right), u_{i}^{-} \in \operatorname{USC}_{p}\left(\mathbb{R}^{N} \times[0, T]\right)$ for $i \in\{1, \ldots, d\}$, is a viscosity subsolution to the problem in (1.1) if $u_{i}^{-}(x, T) \leq g_{i}(x)$ whenever $x \in \mathbb{R}^{N}, i \in$ $\{1, \ldots, d\}$, and if the following holds. If $\left(x_{0}, t_{0}\right) \in \mathbb{R}^{N} \times(0, T)$ and if, for some $i \in\{1, \ldots, d\}$, we have $\phi_{i} \in C^{1,2}\left(\mathbb{R}^{N} \times[0, T)\right)$ such that

(i) $\phi_{i}\left(x_{0}, t_{0}\right)=u_{i}^{-}\left(x_{0}, t_{0}\right)$,

(ii) $\quad\left(x_{0}, t_{0}\right)$ is a global minimum of $\phi_{i}-u_{i}^{-}$, 
then

$$
\min \left\{-\mathcal{H}^{\kappa}\left(\phi_{i}, u_{i}^{-}\right)-\psi_{i}, u_{i}^{-}-\max _{j \neq i}\left(-c_{i, j}+u_{j}^{-}\right)\right\} \leq 0 \text { for all } \kappa \in(0,1) .
$$

(iii) If $\left(u_{1}, \ldots, u_{d}\right)$ is both a viscosity supersolution and subsolution to the problem in (1.1), then $\left(u_{1}, \ldots, u_{d}\right)$ is a viscosity solution to the problem in (1.1).

Remark 2.1. Note that it is natural, since the Levy measure is singular at 0 and has some decay properties at infinity, to break the non-local operator into the pieces $\mathcal{I}_{\kappa}$ and $\mathcal{I}^{\kappa}$ which give, respectively, the contribution from the 'small' and 'large' jumps to $\mathcal{I}$. To have $\mathcal{I}_{\kappa}\left(x, t, v_{i}, D \phi_{i}\right)$ well defined the reasonable things is to choose $v_{i}=\phi_{i}$ in Definition 2. However, for $\mathcal{I}^{\kappa}\left(x, t, v_{i}, D \phi_{i}\right)$ to be well defined the important thing is not regularity of $v_{i}$ but decay properties at infinity of $v_{i}$, decay properties which must be compatible with the decay properties of the Levy measure $\nu$. Hence it is reasonable to use $\mathcal{I}^{\kappa}\left(x, t, v_{i}, D \phi_{i}\right)$ with $v_{i}=u_{i}^{ \pm}$in Definition 2 as long as $u_{i}^{ \pm}$is assumed to have moderated growth at infinity. In our case polynomial growth is sufficient. We refer to [JK06] for an elaboration on this definition and to BI08 for alternative equivalent definitions.

Remark 2.2. In the following we simply write $\mathcal{I}_{\kappa}(\phi, p)=\mathcal{I}_{\kappa}(x, t, \phi, p), \mathcal{I}^{\kappa}(\phi, p)=$ $\mathcal{I}^{\kappa}(x, t, \phi, p)$. Note that

(2.5) $K\left(x, t, z, \phi^{1}+\phi^{2}, p^{1}+p^{2}\right)=K\left(x, t, z, \phi^{1}, p^{1}\right)+K\left(x, t, z, \phi^{2}, p^{2}\right)$

and hence that

$$
\begin{aligned}
& I_{\kappa}\left(\phi^{1}+\phi^{2}, p^{1}+p^{2}\right)=I_{\kappa}\left(\phi^{1}, p^{1}\right)+I_{\kappa}\left(\phi^{2}, p^{2}\right), \\
& I^{\kappa}\left(\phi^{1}+\phi^{2}, p^{1}+p^{2}\right)=I^{\kappa}\left(\phi^{1}, p^{1}\right)+I^{\kappa}\left(\phi^{2}, p^{2}\right) .
\end{aligned}
$$

\section{The COMPARISOn PRINCIPle: PROOF of TheOREM 1.1}

The purpose of this section is to prove Theorem 1.1 and hence through out the section we adopt the assumption stated in Theorem 1.1. In particular, let $\mathcal{H}$ be as in (1.2) and assume (1.8) and (1.9). Assume that $\psi_{i}, c_{i, j}$, and $g_{i}$ are as stated in Theorem 1.1 and assume that $\left(u_{1}^{-}, \ldots, u_{d}^{-}\right)$and $\left(u_{1}^{+}, \ldots, u_{d}^{+}\right)$are viscosity sub- and supersolutions, respectively, to the problem in (1.1). We first prove the following lemma.

Lemma 3.1. The following is true for any $\gamma>0$. Let $\theta \geq 0$. Then there exists $\eta>0$, independent of $\theta$, such that if $\lambda \geq \eta$, then $\left(\bar{u}_{1}^{+}, \ldots, \bar{u}_{d}^{+}\right)$,

$$
\bar{u}_{i}^{+}:=u_{i}^{+}+\theta e^{-\lambda t}\left(|x|^{2 \gamma+2}+1\right) \text { for } i \in\{1, \ldots, d\},
$$

is a viscosity supersolution of (1.1).

Proof. Since $u_{i}^{+} \in \operatorname{LSC}_{p}\left(\mathbb{R}^{N} \times[0, T]\right)$ we have $\bar{u}_{i}^{+} \in \operatorname{LSC}_{p}\left(\mathbb{R}^{N} \times[0, T]\right)$. Let $\left(x_{0}, t_{0}\right) \in$ $\mathbb{R}^{N} \times[0, T]$ and assume, for some $i \in\{1, \ldots, d\}$, that $\phi_{i} \in C^{1,2}\left(\mathbb{R}^{N} \times[0, T)\right)$, satisfies

(i) $\phi_{i}\left(x_{0}, t_{0}\right)=\bar{u}_{i}^{+}\left(x_{0}, t_{0}\right)$,

(ii) $\quad\left(x_{0}, t_{0}\right)$ is a global maximum of $\phi_{i}-\bar{u}_{i}^{+}$.

To prove the lemma it is enough to prove that there exists $\eta>0$, independent of $\theta$, such that if $\lambda \geq \eta$, then

$$
\min \left\{-\mathcal{H}^{\kappa}\left(\phi_{i}, \bar{u}_{i}^{+}\right)-\psi_{i}, \bar{u}_{i}^{+}-\max _{j \neq i}\left(-c_{i, j}+\bar{u}_{j}^{+}\right)\right\} \geq 0 \text { for all } \kappa \in(0,1) .
$$

Let $\Phi_{i}=\phi_{i}-\theta e^{-\lambda t}\left(|x|^{2 \gamma+2}+1\right)$ and note that by construction $\Phi_{i}-u_{i}^{+}$has a global maximum at $\left(x_{0}, t_{0}\right)$. Using that $u_{i}^{+}$is a supersolution we have that

$$
\min \left\{-\mathcal{H}^{\kappa}\left(\Phi_{i}, u_{i}^{+}\right)-\psi_{i}, u_{i}^{+}-\max _{j \neq i}\left(-c_{i, j}+u_{j}^{+}\right)\right\} \geq 0
$$


for all $\kappa \in(0,1)$. Using (3.1) we see that

$$
\bar{u}_{i}^{+}-\max _{j \neq i}\left(-c_{i, j}+\bar{u}_{j}^{+}\right)=u_{i}^{+}-\max _{j \neq i}\left(-c_{i, j}+u_{j}^{+}\right) \geq 0
$$

since $\bar{u}_{i}^{+}-u_{i}^{+}$is independent of $i$. To conclude the proof we hence only have to ensure that

$$
-\mathcal{H}^{\kappa}\left(\phi_{i}, \bar{u}_{i}^{+}\right)-\psi_{i} \geq 0 \text { at }\left(x_{0}, t_{0}\right) \text { and for all } \kappa \in(0,1) \text {. }
$$

Let $\hat{\phi}(x, t)=\theta e^{-\lambda t}\left(|x|^{2 \gamma+2}+1\right)$. Then $\Phi_{i}=\phi_{i}-\hat{\phi}, \bar{u}_{i}^{+}=u_{i}^{+}+\hat{\phi}$. To establish (3.2) we first note, using (3.1), that, at $\left(x_{0}, t_{0}\right)$,

(3.3) $0 \leq-\mathcal{H}^{\kappa}\left(\Phi_{i}, u_{i}^{+}\right)-\psi_{i}=-\mathcal{L} \Phi_{i}-\mathcal{I}_{\kappa}\left(\Phi_{i}, D \Phi_{i}\right)-\mathcal{I}^{\kappa}\left(u_{i}^{+}, D \Phi_{i}\right)-\psi_{i}$.

However, using Remark 2.2 we see that

$$
\begin{aligned}
\mathcal{I}_{\kappa}\left(\Phi_{i}, D \Phi_{i}\right) & =\mathcal{I}_{\kappa}\left(\phi_{i}, D \phi_{i}\right)+\mathcal{I}_{\kappa}(-\hat{\phi},-D \hat{\phi}), \\
\mathcal{I}^{\kappa}\left(u_{i}^{+}, D \Phi_{i}\right) & =\mathcal{I}^{\kappa}\left(\bar{u}_{i}^{+}, D \phi_{i}\right)+\mathcal{I}^{\kappa}(-\hat{\phi},-D \hat{\phi}) .
\end{aligned}
$$

Hence, combining (3.3) and (3.4) we can conclude that

$$
\begin{aligned}
0 \leq & -\mathcal{L} \phi_{i}-\mathcal{I}_{\kappa}\left(\phi_{i}, D \phi_{i}\right)-\mathcal{I}^{\kappa}\left(\bar{u}_{i}^{+}, D \phi_{i}\right)-\psi_{i} \\
& +\mathcal{L} \hat{\phi}-\mathcal{I}_{\kappa}(-\hat{\phi},-D \hat{\phi})-\mathcal{I}_{\kappa}(-\hat{\phi},-D \hat{\phi}) \\
= & -\mathcal{H}^{\kappa}\left(\phi_{i}, \bar{u}_{i}^{+}\right)-\psi_{i}+\mathcal{L} \hat{\phi}-\mathcal{I}(-\hat{\phi},-D \hat{\phi}) .
\end{aligned}
$$

In particular, we have

$$
-\mathcal{H}^{\kappa}\left(\phi_{i}, \bar{u}_{i}^{+}\right)-\psi_{i} \geq-\mathcal{L} \hat{\phi}+\mathcal{I}(-\hat{\phi},-D \hat{\phi}) .
$$

Using this we first note that

$$
-\mathcal{L}(\hat{\phi}) \geq \lambda \theta e^{-\lambda t}\left(|x|^{2 \gamma+2}+1\right)-\mathcal{F}\left(\theta e^{-\lambda t}\left(|x|^{2 \gamma+2}+1\right)\right)
$$

where

$$
\mathcal{F}:=\sum_{i, j=1}^{N} a_{i j}(x, t) \partial_{x_{i} x_{j}}+\sum_{i=1}^{N} a_{i}(x, t) \partial_{x_{i}} .
$$

Using the assumption on the operator $\mathcal{H}$ stated in Theorem 1.1 we see that

$$
\left|\mathcal{F}\left(\theta e^{-\lambda t}\left(|x|^{2 \gamma+2}+1\right)\right)\right| \leq c \theta e^{-\lambda t}\left(|x|^{2 \gamma+2}+1\right)
$$

and hence

$$
-\mathcal{L}(\hat{\phi}) \geq(\lambda-c) \hat{\phi} .
$$

To estimate $\mathcal{I}(-\hat{\phi},-D \hat{\phi})$ we first note, by definition, that

$$
\begin{aligned}
\mathcal{I}(x, t,-\hat{\phi},-D \hat{\phi})= & -\int_{\mathbb{R}^{l} \backslash\{0\}} K(x, t, z, \hat{\phi}, D \hat{\phi}) \nu(d z), \\
K(x, t, z, \hat{\phi}, D \hat{\phi})= & \hat{\phi}(x+\eta(x, t, z), t)-\hat{\phi}(x, t) \\
& -\chi_{\{|z| \leq 1\}}(z) \sum_{k=1}^{N} \eta_{k}(x, t, z) \partial_{x_{k}} \hat{\phi} .
\end{aligned}
$$

Hence,

$$
\begin{aligned}
|\mathcal{I}(x, t,-\hat{\phi},-D \hat{\phi})| \leq & \int_{B(0,1) \backslash\{0\}}|K(x, t, z, \hat{\phi}, D \hat{\phi})| \nu(d z), \\
& +\int_{\mathbb{R}^{l} \backslash B(0,1)}|K(x, t, z, \hat{\phi}, D \hat{\phi})| \nu(d z) \\
= & T_{1}+T_{2} .
\end{aligned}
$$

Now, using (1.11), and Taylor's formula, we first see that

$$
|K(x, t, z, \hat{\phi}, D \hat{\phi})| \leq c|z|^{2} \hat{\phi}(x, t) \quad \text { whenever } z \in B(0,1) \backslash\{0\},
$$




$$
|K(x, t, z, \hat{\phi}, D \hat{\phi})| \leq c \hat{\phi}(x, t) \quad \text { whenever } z \in \mathbb{R}^{l} \backslash B(0,1) .
$$

Hence, using this, (1.10), and (1.11) we can conclude that

$$
T_{1} \leq c \hat{\phi}(x, t) \text { and } T_{2} \leq c \hat{\phi}(x, t) .
$$

Putting the estimates together we can conclude, at $\left(x_{0}, t_{0}\right)$ and for all $\kappa \in(0,1)$, that

$$
-\mathcal{H}^{\kappa}\left(\phi_{i}, \bar{u}_{i}^{+}\right)-\psi_{i} \geq-\mathcal{L} \hat{\phi}+\mathcal{I}(-\hat{\phi},-D \hat{\phi}) \geq(\lambda-c) \hat{\phi}\left(x_{0}, t_{0}\right) .
$$

In view of (3.2) we see that (3.9) completes the proof of the lemma.

3.1. Proof of Theorem 1.1. Assume that $\left(u_{1}^{+}, \ldots, u_{d}^{+}\right)$is a viscosity supersolution and that $\left(u_{1}^{-}, \ldots, u_{d}^{-}\right)$is a viscosity subsolution, respectively, to the problem in (1.1). We want to prove that

$$
u_{i}^{-}(x, t) \leq u_{i}^{+}(x, t), \text { for all } i \in\{1, \ldots, d\},
$$

whenever $(x, t) \in \mathbb{R}^{N} \times(0, T]$. In fact, we will prove a slightly modified version of (3.10). We let $\tilde{u}_{i}^{-}(x, t)=e^{t} u_{i}^{-}(x, t)$ and $\tilde{u}_{i}^{+}(x, t)=e^{t} \bar{u}_{i}^{+}(x, t)$ for all $i \in\{1, \ldots, d\}$. One can easily verify that $\left(\tilde{u}_{1}^{-}, \ldots, \tilde{u}_{d}^{-}\right)$is a viscosity subsolution to the problem

$$
\min \left\{\tilde{u}_{i}^{-}-\mathcal{H} \tilde{u}_{i}^{-}(x, t)-\tilde{\psi}_{i}(x, t), \tilde{u}_{i}^{-}(x, t)-\max _{j \neq i}\left(-\tilde{c}_{i, j}(x, t)+\tilde{u}_{j}^{-}(x, t)\right)\right\}=0,
$$

(3.11) $\tilde{u}_{i}^{-}(x, T)=\tilde{g}_{i}(x)$,

where

$$
\tilde{\psi}_{i}(x, t)=e^{t} \psi_{i}(x, t), \tilde{c}_{i, j}(x, t)=e^{t} c_{i, j}(x, t), \tilde{g}_{i}(x)=e^{T} g_{i}(x),
$$

if and only if $\left(u_{1}^{-}, \ldots, u_{d}^{-}\right)$is a subsolution to (1.1). Similarly, $\left(\tilde{u}_{1}^{+}, \ldots, \tilde{u}_{d}^{+}\right)$is a viscosity supersolution to the problem in (3.11) if and only if $\left(u_{1}^{+}, \ldots, u_{d}^{+}\right)$is a supersolution to (1.1). Proving (3.10) is now equivalent to proving

$$
\tilde{u}_{i}^{-}(x, t) \leq \tilde{u}_{i}^{+}(x, t), \text { for all } i \in\{1, \ldots, d\},
$$

whenever $(x, t) \in \mathbb{R}^{N} \times(0, T]$. However, according to Lemma 3.1 it is enough to prove

$$
\tilde{u}_{i}^{-}(x, t) \leq \bar{u}_{i}^{+}(x, t) \text {, for all } i \in\{1, \ldots, d\},
$$

whenever $(x, t) \in \mathbb{R}^{N} \times(0, T]$, where $\bar{u}_{i}^{+}:=\tilde{u}_{i}^{+}+\theta e^{-(\lambda-1) t}\left(|x|^{2 \gamma+2}+1\right), \gamma>0$, since we easily recover (3.12) by letting $\theta \rightarrow 0$ in (3.13). In fact, it is enough to prove that

$$
\tilde{u}_{i}^{-}(x, t) \leq \bar{u}_{i}^{+}(x, t)+\frac{\theta}{t}, \text { for all } i \in\{1, \ldots, d\},
$$

whenever $(x, t) \in \mathbb{R}^{N} \times(0, T]$ and for any $\theta>0$, since the desired result is still retrieved in the limit as $\theta \rightarrow 0$. In particular, we will prove (3.14) for $\theta$ fixed and then let $\theta \rightarrow 0$.

Let in the following $B(0, R), R>0$, be the standard Euclidean ball of radius $R$ centered at 0 . Using that $u_{i}^{-} \in \mathrm{USC}_{p}\left(\mathbb{R}^{N} \times[0, T]\right)$ for $i \in\{1, \ldots, d\}$, we have that there exists $\gamma_{0} \geq \frac{1}{2}$ such that $\left|\tilde{u}_{i}^{-}(x, t)\right| \leq c\left(1+|x|^{2 \gamma_{0}}\right)$. We now fix this $\gamma_{0}$ and plug it into our defintion of $\bar{u}_{i}^{+}(x, t)$ from above. With slight abuse of notation, we will drop the subscript from $\gamma_{0}$ and only write $\gamma$ and let $\bar{u}_{i}^{+}(x, t)$ from here on in denote $\tilde{u}_{i}^{+}+\theta e^{-(\lambda-1) t}\left(|x|^{2 \gamma+2}+1\right)$ with $\gamma=\gamma_{0}$ fixed. Using this $\tilde{u}_{i}^{+}$we see that there exists $R>0$ such that

(3.15) $\tilde{u}_{i}^{-}(x, t)-\bar{u}_{i}^{+}(x, t)<0$ whenever $(x, t) \in\left(\mathbb{R}^{N} \backslash B(0, R)\right) \times[0, T]$. 
From 3.15 we see that

$$
\begin{aligned}
& \sup _{(x, t) \in \mathbb{R}^{N} \times[0, T]} \max _{i \in\{1, \ldots, d\}}\left(\left(\tilde{u}_{i}^{-}(x, t)-\bar{u}_{i}^{+}(x, t)-\frac{\theta}{t}\right)\right. \\
= & \sup _{(x, t) \in B(0, R) \times[0, T]} \max _{i \in\{1, \ldots, d\}}\left(\left(\tilde{u}_{i}^{-}(x, t)-\bar{u}_{i}^{+}(x, t)-\frac{\theta}{t}\right)\right. \\
= & \max _{i \in\{1, \ldots, d\}}\left(\left(\tilde{u}_{i}^{-}(\bar{x}, \bar{t})-\bar{u}_{i}^{+}(\bar{x}, \bar{t})-\frac{\theta}{\bar{t}}\right)\right.
\end{aligned}
$$

for some $(\bar{x}, \bar{t}) \in B(0, R) \times(0, T]$. We will now prove (3.14) by contradiction. Indeed, assume that

$$
\max _{i \in\{1, \ldots, d\}}\left(\tilde{u}_{i}^{-}(\bar{x}, \bar{t})-\bar{u}_{i}^{+}(\bar{x}, \bar{t})-\frac{\theta}{\bar{t}}\right)>0 .
$$

Using that $\tilde{u}_{i}^{-}(x, T) \leq \bar{u}_{i}^{+}(x, T)$, for all $x \in \mathbb{R}^{N}$, by the definition of sub- and supersolution, we see that $(\bar{x}, \bar{t}) \in B(0, R) \times(0, T)$. For $(\bar{x}, \bar{t}) \in B(0, R) \times(0, T)$ fixed, we let $\mathcal{I}$ be the non-empty set of all $j \in\{1, \ldots, d\}$ such that

$$
\left(\tilde{u}_{j}^{-}(\bar{x}, \bar{t})-\bar{u}_{j}^{+}(\bar{x}, \bar{t})-\frac{\theta}{\bar{t}}\right)=\max _{i \in\{1, \ldots, d\}}\left(\tilde{u}_{i}^{-}(\bar{x}, \bar{t})-\bar{u}_{i}^{+}(\bar{x}, \bar{t})-\frac{\theta}{\bar{t}}\right) .
$$

For $\gamma=1$ and for given degrees of freedom $\beta>0, \Lambda>0$, we introduce the function $\varphi_{\varepsilon}: \mathbb{R}^{N} \times \mathbb{R}^{N} \times[0, T] \rightarrow \mathbb{R}$,

$$
\varphi_{\varepsilon}(x, y, t)=\frac{1}{2 \varepsilon}|x-y|^{2}+\Lambda\left(|x-\bar{x}|^{2 \gamma+2}+|y-\bar{x}|^{2 \gamma+2}\right)+\beta(t-\bar{t})^{2} .
$$

Note that $\varphi_{\varepsilon}$ is non-negative. Furthermore, for $j \in \mathcal{I}$ fixed, and $\varepsilon, 0<\varepsilon \ll 1$, we consider the function

$$
\phi_{\varepsilon}^{j}(x, y, t)=\tilde{u}_{j}^{-}(x, t)-\bar{u}_{j}^{+}(y, t)-\frac{\theta}{t}-\varphi_{\varepsilon}(x, y, t),
$$

where $(x, y, t) \in B(0, R) \times B(0, R) \times[0, T]$. Using that $\tilde{u}_{j}^{-}$is upper semi-continuous and that $\bar{u}_{j}^{+}$is lower semi-continuous we can conclude that $\phi_{\varepsilon}^{j}$ is upper semicontinuous and hence that there exist $\left(x_{\varepsilon}, y_{\varepsilon}, t_{\varepsilon}\right)$ such that

$$
\phi_{\varepsilon}^{j}\left(x_{\varepsilon}, y_{\varepsilon}, t_{\varepsilon}\right)=\sup _{(x, y, t) \in \overline{B(0, R)} \times \overline{B(0, R)} \times[0, T]} \phi_{\varepsilon}^{j}(x, y, t) .
$$

Note that the points $\left(x_{\varepsilon}, y_{\varepsilon}, t_{\varepsilon}\right)$ depend also on $\beta$ and $\Lambda$. However, in this part of the argument $\beta$ and $\Lambda$ are kept fixed and hence the dependence is harmless in the following. Using that $2 \phi^{j}\left(x_{\varepsilon}, y_{\varepsilon}, t_{\varepsilon}\right) \geq \phi^{j}\left(x_{\varepsilon}, x_{\varepsilon}, t_{\varepsilon}\right)+\phi^{j}\left(y_{\varepsilon}, y_{\varepsilon}, t_{\varepsilon}\right)$ we see that

$$
\frac{1}{\varepsilon}\left|x_{\varepsilon}-y_{\varepsilon}\right|^{2} \leq \tilde{u}_{j}^{-}\left(x_{\varepsilon}, t_{\varepsilon}\right)-\tilde{u}_{j}^{-}\left(y_{\varepsilon}, t_{\varepsilon}\right)+\bar{u}_{j}^{+}\left(x_{\varepsilon}, t_{\varepsilon}\right)-\bar{u}_{j}^{+}\left(y_{\varepsilon}, t_{\varepsilon}\right),
$$

and as the right hand side of (3.19) is bounded we have that $\left|x_{\varepsilon}-y_{\varepsilon}\right| \rightarrow 0$ as $\varepsilon \rightarrow 0$. Using that $(\bar{x}, \bar{t}) \in \overline{B(0, R)} \times(0, T)$, and the construction of $\varphi_{\varepsilon}$, we see that

$$
\begin{aligned}
\tilde{u}_{j}^{-}(\bar{x}, \bar{t})-\bar{u}_{j}^{+}(\bar{x}, \bar{t})-\frac{\theta}{\bar{t}} & =\phi_{\varepsilon}^{j}(\bar{x}, \bar{x}, \bar{t}) \\
& \leq \phi_{\varepsilon}^{j}\left(x_{\varepsilon}, y_{\varepsilon}, t_{\varepsilon}\right) \leq \tilde{u}_{j}^{-}\left(x_{\varepsilon}, t_{\varepsilon}\right)-\bar{u}_{j}^{+}\left(y_{\varepsilon}, t_{\varepsilon}\right)-\frac{\theta}{t_{\varepsilon}} .
\end{aligned}
$$

Furthermore, we see that we must have, using the definition of $(\bar{x}, \bar{t}),\left(x_{\varepsilon}, y_{\varepsilon}, t_{\varepsilon}\right)$, and the upper semi-continuity of $\tilde{u}_{j}^{-}-\bar{u}_{j}^{+}$, that

$$
\left(x_{\varepsilon}, y_{\varepsilon}, t_{\varepsilon}\right) \rightarrow(\bar{x}, \bar{x}, \bar{t}) \text { as } \varepsilon \rightarrow 0 .
$$

The above display also shows that, for $\varepsilon$ small enough, we have $t_{\varepsilon} \in(0, T)$ since $t_{\varepsilon} \rightarrow \bar{t}$ and $\bar{t} \in(0, T)$. Note also that

$$
\tilde{u}_{j}^{-}\left(x_{\varepsilon}, t_{\varepsilon}\right) \rightarrow \tilde{u}_{j}^{-}(\bar{x}, \bar{t}) \text { and } \bar{u}_{j}^{+}\left(y_{\varepsilon}, t_{\varepsilon}\right) \rightarrow \bar{u}_{j}^{+}(\bar{x}, \bar{t}) \text { as } \varepsilon \rightarrow 0 .
$$


Indeed, recall that $\tilde{u}_{j}^{-}$is upper semi-continuous and assume, taking (3.21) into account, that $\lim \sup _{\varepsilon \rightarrow 0} \tilde{u}_{j}^{-}\left(x_{\varepsilon}, t_{\varepsilon}\right)<\tilde{u}_{j}^{-}(\bar{x}, \bar{t})$. Then, using (3.20) we have that $\liminf _{\varepsilon \rightarrow 0} \bar{u}_{j}^{+}\left(y_{\varepsilon}, t_{\varepsilon}\right)<\bar{u}_{j}^{+}(\bar{x}, \bar{t})$ but this contradicts the lower semi-continuity for $\tilde{u}_{j}^{+}$. Similarly, assuming that $\liminf _{\varepsilon \rightarrow 0} \bar{u}_{j}^{+}\left(y_{\varepsilon}, t_{\varepsilon}\right)>\bar{u}_{j}^{+}(\bar{x}, \bar{t})$ we see that

$$
\limsup _{\varepsilon \rightarrow 0} \tilde{u}_{j}^{-}\left(x_{\varepsilon}, t_{\varepsilon}\right)>\tilde{u}_{j}^{-}(\bar{x}, \bar{t}),
$$

which again is a contradiction. Repeating (3.20) we also have that

$$
\begin{aligned}
& \tilde{u}_{j}^{-}(\bar{x}, \bar{t})-\bar{u}_{j}^{+}(\bar{x}, \bar{t})-\frac{\theta}{\bar{t}}=\phi_{\varepsilon}^{j}(\bar{x}, \bar{x}, \bar{t}) \\
\leq & \phi_{\varepsilon}^{j}\left(x_{\varepsilon}, y_{\varepsilon}, t_{\varepsilon}\right)=\tilde{u}_{j}^{-}\left(x_{\varepsilon}, t_{\varepsilon}\right)-\bar{u}_{j}^{+}\left(y_{\varepsilon}, t_{\varepsilon}\right)-\varphi_{\varepsilon}\left(x_{\varepsilon}, y_{\varepsilon}, t_{\varepsilon}\right)-\frac{\theta}{t_{\varepsilon}} .
\end{aligned}
$$

In particular,

$$
\begin{aligned}
\varphi_{\varepsilon}\left(x_{\varepsilon}, y_{\varepsilon}, t_{\varepsilon}\right) \leq & \tilde{u}_{j}^{-}\left(x_{\varepsilon}, t_{\varepsilon}\right)-\tilde{u}_{j}^{-}(\bar{x}, \bar{t}) \\
& +\bar{u}_{j}^{+}(\bar{x}, \bar{t})-\bar{u}_{j}^{+}\left(y_{\varepsilon}, t_{\varepsilon}\right)+\frac{\theta}{\bar{t}}-\frac{\theta}{t_{\varepsilon}}
\end{aligned}
$$

and using (3.22) we see that

$$
\lim _{\varepsilon \rightarrow 0} \varphi_{\varepsilon}\left(x_{\varepsilon}, y_{\varepsilon}, t_{\varepsilon}\right)=0 .
$$

In particular,

$$
\frac{1}{\varepsilon}\left|x_{\varepsilon}-y_{\varepsilon}\right|^{2} \rightarrow 0 \text { as } \varepsilon \rightarrow 0 .
$$

To proceed we will now argue as in [K91, using the no-loop condition (1.13) (ii), to conclude that there exists $k \in \mathcal{I}$ such that

$$
\tilde{u}_{k}^{-}(\bar{x}, \bar{t})>\max _{i \in\{1, \ldots, k-1, k+1, \ldots, d\}}\left(\tilde{u}_{i}^{-}(\bar{x}, \bar{t})-\tilde{c}_{k, i}(\bar{x}, \bar{t})\right) .
$$

Indeed, assume, on the contrary, that

$$
\tilde{u}_{k}^{-}(\bar{x}, \bar{t}) \leq \max _{i \in\{1, \ldots, k-1, k+1, \ldots, d\}}\left(\tilde{u}_{i}^{-}(\bar{x}, \bar{t})-\tilde{c}_{k, i}(\bar{x}, \bar{t})\right)
$$

for all $k \in \mathcal{I}$ and hence, in particular, that

$$
\tilde{u}_{k}^{-}(\bar{x}, \bar{t})+\tilde{c}_{k, j}(\bar{x}, \bar{t}) \leq \tilde{u}_{j}^{-}(\bar{x}, \bar{t})
$$

for some $j \in\{1, \ldots, k-1, k+1, \ldots, d\}$. Furthermore, since $\left(\bar{u}_{1}^{+}, \ldots, \bar{u}_{d}^{+}\right)$is a supersolution to (3.11) we have that

$$
\bar{u}_{k}^{+}(\bar{x}, \bar{t}) \geq \bar{u}_{j}^{+}(\bar{x}, \bar{t})-\tilde{c}_{k, j}(\bar{x}, \bar{t}) .
$$

Combining the two inequalities above yields

$$
\tilde{u}_{k}^{-}(\bar{x}, \bar{t})-\tilde{u}_{j}^{-}(\bar{x}, \bar{t}) \leq-\tilde{c}_{k, j}(\bar{x}, \bar{t}) \leq \bar{u}_{k}^{+}(\bar{x}, \bar{t})-\bar{u}_{j}^{+}(\bar{x}, \bar{t}),
$$

and hence

$$
\tilde{u}_{k}^{-}(\bar{x}, \bar{t})-\bar{u}_{k}^{+}(\bar{x}, \bar{t})-\frac{\theta}{\bar{t}} \leq \tilde{u}_{j}^{-}(\bar{x}, \bar{t})-\bar{u}_{j}^{+}(\bar{x}, \bar{t})-\frac{\theta}{\bar{t}} .
$$

But $k \in \mathcal{I}$ so (3.29) is actually an equality and hence $j \in \mathcal{I}$. Repeating this argument as many times as necessary we get the existence of a loop of indices $\left\{i_{1}, i_{2}, \ldots, i_{p}, i_{p+1}\right\}$ such that $i_{1}=i_{p+1}$ and

$$
\tilde{c}_{i_{1}, i_{2}}+\tilde{c}_{i_{2}, i_{3}}+\cdots+\tilde{c}_{i_{p}, i_{p+1}}=0 .
$$

This contradicts our assumptions on the switching costs and hence (3.27) must hold. 
We now consider $k \in \mathcal{I}$ such that 3.27 holds and we intend to derive a contradiction to the assumption in (3.17). First, using (3.22) and (3.27) we see that there exists $\bar{\varepsilon}, 0<\bar{\varepsilon} \ll 1$, such that

$$
\tilde{u}_{k}^{-}\left(x_{\varepsilon}, t_{\varepsilon}\right)>\max _{i \in\{1, \ldots, k-1, k+1, \ldots, d\}}\left(\tilde{u}_{i}^{-}\left(x_{\varepsilon}, t_{\varepsilon}\right)-\tilde{c}_{k, i}\left(x_{\varepsilon}, t_{\varepsilon}\right)\right) \text { when } 0<\varepsilon<\bar{\varepsilon} .
$$

(3.31) ensures that $\tilde{u}_{k}^{-}$is above the obstacle at the points $\left\{\left(x_{\varepsilon}, t_{\varepsilon}\right)\right\}_{\varepsilon<\bar{\varepsilon}}$. We next intend to apply the so called maximum principle for semi-continuous functions, in our case adapted to the non-local system, in a neighborhood of $\left(x_{\varepsilon}, y_{\varepsilon}, t_{\varepsilon}\right)$ and for $\tilde{u}_{k}^{-}$, and along the lines of [BI08]. To do this we first have to calculate $\partial_{t} \varphi_{\varepsilon}, \partial_{x_{i}} \varphi_{\varepsilon}$, $\partial_{y_{i}} \varphi_{\varepsilon}$ and $\partial_{x_{i} y_{j}} \varphi_{\varepsilon}$. Doing this we see that

$$
\begin{aligned}
& \partial_{t} \varphi_{\varepsilon}(x, y, t)=2 \beta(t-\bar{t}), \\
& D_{x} \varphi_{\varepsilon}(x, y, t)=\frac{1}{\varepsilon}(x-y)+\Lambda(2 \gamma+2)(x-\bar{x})|x-\bar{x}|^{2 \gamma} \\
& D_{y} \varphi_{\varepsilon}(x, y, t)=-\frac{1}{\varepsilon}(x-y)+\Lambda(2 \gamma+2)(y-\bar{x})|y-\bar{x}|^{2 \gamma} .
\end{aligned}
$$

Furthermore,

$$
D_{x, y}^{2} \varphi_{\varepsilon}(x, y)=\frac{1}{\varepsilon}\left(\begin{array}{cc}
I & -I \\
-I & I
\end{array}\right)+\left(\begin{array}{cc}
l(x) & 0 \\
0 & l(y)
\end{array}\right)
$$

where

$$
l(x)=\Lambda(2 \gamma+2)|x-\bar{x}|^{2 \gamma} I+2 \gamma \Lambda(2 \gamma+2)\langle x-\bar{x}, x-\bar{x}\rangle|x-\bar{x}|^{2 \gamma-2} .
$$

Let $S_{N}$ be the set of all $N \times N$-dimensional symmetric matrices and let $\varphi_{\varepsilon}^{\alpha}$ be the sup-convolution of $\varphi_{\varepsilon}$ as defined in BI08]. We may now apply Lemma 1 of [BI08] (more precisely Corollary 2) to conclude that for any $\kappa>0$ there exists $\bar{\alpha}(\kappa)$ such that for $0<\alpha \leq \bar{\alpha}$ there exist $C, D \in \mathbb{R}$ and $X, Y \in S_{N}$, such that

$$
\begin{gathered}
C+D=2 \beta\left(t_{\varepsilon}-\bar{t}\right)-\frac{\theta}{t_{\varepsilon}^{2}} \\
\left(\begin{array}{cc}
X & 0 \\
0 & -Y
\end{array}\right) \leq D_{x, y}^{2} \varphi_{\varepsilon}\left(x_{\varepsilon}, y_{\varepsilon}\right)+o_{\alpha}(1)\left(\begin{array}{cc}
I & 0 \\
0 & I
\end{array}\right),
\end{gathered}
$$

and such that

$$
\begin{aligned}
& -C+\tilde{u}_{k}^{-}\left(x_{\varepsilon}, t_{\varepsilon}\right)-\sum_{i, j=1}^{N} a_{i j}\left(x_{\varepsilon}, t_{\varepsilon}\right) X_{i, j}-\sum_{i=1}^{N} a_{i}\left(x_{\varepsilon}, t_{\varepsilon}\right) \partial_{x_{i}} \varphi_{\varepsilon}\left(x_{\varepsilon}, y_{\varepsilon}, t_{\varepsilon}\right) \\
& -\mathcal{I}_{\kappa}\left(x_{\varepsilon}, t_{\varepsilon}, \varphi_{\varepsilon}^{\alpha}\left(\cdot, y_{\varepsilon}, t_{\varepsilon}\right), D_{x} \varphi_{\varepsilon}\right) \\
& -\mathcal{I}^{\kappa}\left(x_{\varepsilon}, t_{\varepsilon}, \tilde{u}_{k}^{-}\left(\cdot, t_{\varepsilon}\right), D_{x} \varphi_{\varepsilon}\right)-\tilde{\psi}_{k}\left(x_{\varepsilon}, t_{\varepsilon}\right) \leq 0
\end{aligned}
$$

and

$$
\begin{aligned}
& D+\bar{u}_{k}^{+}\left(y_{\varepsilon}, t_{\varepsilon}\right)-\sum_{i, j=1}^{N} a_{i j}\left(y_{\varepsilon}, t_{\varepsilon}\right) Y_{i, j}+\sum_{i=1}^{N} a_{i}\left(y_{\varepsilon}, t_{\varepsilon}\right) \partial_{y_{i}} \varphi_{\varepsilon}\left(x_{\varepsilon}, y_{\varepsilon}, t_{\varepsilon}\right) \\
& -\mathcal{I}_{\kappa}\left(y_{\varepsilon}, t_{\varepsilon},-\varphi_{\varepsilon}^{\alpha}\left(x_{\varepsilon}, \cdot t_{\varepsilon}\right),-D_{y} \varphi_{\varepsilon}\right) \\
& -\mathcal{I}^{\kappa}\left(y_{\varepsilon}, t_{\varepsilon}, \bar{u}_{k}^{+}\left(\cdot, t_{\varepsilon}\right),-D_{y} \varphi_{\varepsilon}\right)-\tilde{\psi}_{k}\left(y_{\varepsilon}, t_{\varepsilon}\right) \geq 0 .
\end{aligned}
$$

In the above, we have used little o notation, i.e., $o_{\alpha}(1) \rightarrow 0$ as $\alpha \rightarrow 0$. Adding (3.35) and (3.36) we see that

$$
-C-D+\tilde{u}_{k}^{-}\left(x_{\varepsilon}, t_{\varepsilon}\right)-\bar{u}_{k}^{+}\left(y_{\varepsilon}, t_{\varepsilon}\right)-\sum_{i, j=1}^{N}\left(a_{i j}\left(x_{\varepsilon}, t_{\varepsilon}\right) X_{i, j}-a_{i j}\left(y_{\varepsilon}, t_{\varepsilon}\right) Y_{i, j}\right)
$$




$$
\begin{aligned}
& -\sum_{i=1}^{N}\left(a_{i}\left(x_{\varepsilon}, t_{\varepsilon}\right) \partial_{x_{i}} \varphi_{\varepsilon}\left(x_{\varepsilon}, y_{\varepsilon}, t_{\varepsilon}\right)+a_{i}\left(y_{\varepsilon}, t_{\varepsilon}\right) \partial_{y_{i}} \varphi_{\varepsilon}\left(x_{\varepsilon}, y_{\varepsilon}, t_{\varepsilon}\right)\right) \\
& -\left(\tilde{\psi}_{k}\left(x_{\varepsilon}, t_{\varepsilon}\right)-\tilde{\psi}_{k}\left(y_{\varepsilon}, t_{\varepsilon}\right)\right) \\
& -\left(\mathcal{I}_{\kappa}\left(x_{\varepsilon}, t_{\varepsilon}, \varphi_{\varepsilon}^{\alpha}\left(\cdot, y_{\varepsilon}, t_{\varepsilon}\right), D_{x} \varphi_{\varepsilon}\right)-\mathcal{I}_{\kappa}\left(x_{\varepsilon}, t_{\varepsilon},-\varphi_{\varepsilon}^{\alpha}\left(x_{\varepsilon}, \cdot, t_{\varepsilon}\right),-D_{y} \varphi_{\varepsilon}\right)\right) \\
& -\left(\mathcal{I}^{\kappa}\left(y_{\varepsilon}, t_{\varepsilon}, \tilde{u}_{k}^{-}\left(\cdot, t_{\varepsilon}\right), D_{x} \varphi_{\varepsilon}\right)-\mathcal{I}^{\kappa}\left(y_{\varepsilon}, t_{\varepsilon}, \bar{u}_{k}^{+}\left(\cdot, t_{\varepsilon}\right),-D_{y} \varphi_{\varepsilon}\right)\right) \leq 0 .
\end{aligned}
$$

Now, using standard arguments based on assumptions (1.8) and (1.9) it follows that

$$
\begin{aligned}
& \left|\sum_{i=1}^{N}\left(a_{i}\left(x_{\varepsilon}, t_{\varepsilon}\right) \partial_{x_{i}} \varphi_{\varepsilon}\left(x_{\varepsilon}, y_{\varepsilon}, t_{\varepsilon}\right)+a_{i}\left(y_{\varepsilon}, t_{\varepsilon}\right) \partial_{y_{i}} \varphi_{\varepsilon}\left(x_{\varepsilon}, y_{\varepsilon}, t_{\varepsilon}\right)\right)\right| \\
\leq & c\left(\frac{1}{\varepsilon}\left|x_{\varepsilon}-y_{\varepsilon}\right|^{2}+\Lambda\left(1+\left|x_{\varepsilon}\right|\left|x_{\varepsilon}-\bar{x}\right|^{2 \gamma+1}+\left|y_{\varepsilon}\right|\left|y_{\varepsilon}-\bar{x}\right|^{2 \gamma+1}\right)\right)
\end{aligned}
$$

and

$$
\begin{aligned}
& \left|\sum_{i, j=1}^{N}\left(a_{i j}\left(x_{\varepsilon}, t_{\varepsilon}\right) X_{i, j}-a_{i j}\left(y_{\varepsilon}, t_{\varepsilon}\right) Y_{i, j}\right)\right| \\
\leq & c\left(\frac{1}{\varepsilon}\left|x_{\varepsilon}-y_{\varepsilon}\right|^{2}+\Lambda\left(1+\left|x_{\varepsilon}\right|^{2}\left|x_{\varepsilon}-\bar{x}\right|^{2 \gamma}+\left|y_{\varepsilon}\right|^{2}\left|y_{\varepsilon}-\bar{x}\right|^{2 \gamma}\right)\right) .
\end{aligned}
$$

Putting these estimates together we find that

$$
\begin{aligned}
& -C-D+\tilde{u}_{k}^{-}\left(x_{\varepsilon}, t_{\varepsilon}\right)-\bar{u}_{k}^{+}\left(y_{\varepsilon}, t_{\varepsilon}\right) \\
& -\left(\mathcal{I}_{\kappa}\left(x_{\varepsilon}, t_{\varepsilon}, \varphi_{\varepsilon}^{\alpha}\left(\cdot, y_{\varepsilon}, t_{\varepsilon}\right), D_{x} \varphi_{\varepsilon}\right)-\mathcal{I}_{\kappa}\left(x_{\varepsilon}, t_{\varepsilon},-\varphi_{\varepsilon}^{\alpha}\left(x_{\varepsilon}, \cdot, t_{\varepsilon}\right),-D_{y} \varphi_{\varepsilon}\right)\right) \\
& -\left(\mathcal{I}^{\kappa}\left(y_{\varepsilon}, t_{\varepsilon}, \tilde{u}_{k}^{-}\left(\cdot, t_{\varepsilon}\right), D_{x} \varphi_{\varepsilon}\right)-\mathcal{I}^{\kappa}\left(y_{\varepsilon}, t_{\varepsilon}, \bar{u}_{k}^{+}\left(\cdot, t_{\varepsilon}\right),-D_{y} \varphi_{\varepsilon}\right)\right) \\
\leq & \frac{c}{\varepsilon}\left|x_{\varepsilon}-y_{\varepsilon}\right|^{2}+c \Lambda h\left(x_{\varepsilon}, y_{\varepsilon}, \bar{x}, \bar{y}\right)+\tilde{\psi}_{k}\left(x_{\varepsilon}, t_{\varepsilon}\right)-\tilde{\psi}_{k}\left(y_{\varepsilon}, t_{\varepsilon}\right)
\end{aligned}
$$

where $0 \leq h\left(x_{\varepsilon}, y_{\varepsilon}, \bar{x}, \bar{y}\right) \leq c$ for all $\varepsilon, 0<\varepsilon \leq \bar{\varepsilon}$. Hence, using the relation for $C+D$, see (3.34) we see that

$$
\begin{aligned}
& \tilde{u}_{k}^{-}\left(x_{\varepsilon}, t_{\varepsilon}\right)-\bar{u}_{k}^{+}\left(y_{\varepsilon}, t_{\varepsilon}\right)-2 \beta\left(t_{\varepsilon}-\bar{t}\right)+\frac{\theta}{t_{\varepsilon}^{2}} \\
& -\left(\mathcal{I}_{\kappa}\left(x_{\varepsilon}, t_{\varepsilon}, \varphi_{\varepsilon}^{\alpha}\left(\cdot, y_{\varepsilon}, t_{\varepsilon}\right), D_{x} \varphi_{\varepsilon}\right)-\mathcal{I}_{\kappa}\left(x_{\varepsilon}, t_{\varepsilon},-\varphi_{\varepsilon}^{\alpha}\left(x_{\varepsilon}, \cdot, t_{\varepsilon}\right),-D_{y} \varphi_{\varepsilon}\right)\right) \\
& -\left(\mathcal{I}^{\kappa}\left(y_{\varepsilon}, t_{\varepsilon}, \tilde{u}_{k}^{-}\left(\cdot, t_{\varepsilon}\right), D_{x} \varphi_{\varepsilon}\right)-\mathcal{I}^{\kappa}\left(y_{\varepsilon}, t_{\varepsilon}, \bar{u}_{k}^{+}\left(\cdot, t_{\varepsilon}\right),-D_{y} \varphi_{\varepsilon}\right)\right) \\
\leq & \frac{c}{\varepsilon}\left|x_{\varepsilon}-y_{\varepsilon}\right|^{2}+c \Lambda h\left(x_{\varepsilon}, y_{\varepsilon}, \bar{x}, \bar{y}\right)+\tilde{\psi}_{k}\left(x_{\varepsilon}, t_{\varepsilon}\right)-\tilde{\psi}_{k}\left(y_{\varepsilon}, t_{\varepsilon}\right) .
\end{aligned}
$$

We now need to estimate the expressions involving the non-local operators and we intend to prove that

$$
\begin{aligned}
& \mid-\left(\mathcal{I}_{\kappa}\left(x_{\varepsilon}, t_{\varepsilon}, \varphi_{\varepsilon}^{\alpha}\left(\cdot, y_{\varepsilon}, t_{\varepsilon}\right), D_{x} \varphi_{\varepsilon}\right)-\mathcal{I}_{\kappa}\left(x_{\varepsilon}, t_{\varepsilon},-\varphi_{\varepsilon}^{\alpha}\left(x_{\varepsilon}, \cdot, t_{\varepsilon}\right),-D_{y} \varphi_{\varepsilon}\right)\right) \\
& -\left(\mathcal{I}^{\kappa}\left(y_{\varepsilon}, t_{\varepsilon}, \tilde{u}_{k}^{-}\left(\cdot, t_{\varepsilon}\right), D_{x} \varphi_{\varepsilon}\right)-\mathcal{I}^{\kappa}\left(y_{\varepsilon}, t_{\varepsilon}, \bar{u}_{k}^{+}\left(\cdot, t_{\varepsilon}\right),-D_{y} \varphi_{\varepsilon}\right)\right) \mid
\end{aligned}
$$

$(3.38) \leq \frac{c}{\varepsilon}\left|x_{\varepsilon}-y_{\varepsilon}\right|^{2}+c \Lambda \hat{h}\left(x_{\varepsilon}, y_{\varepsilon}, \bar{x}, \bar{y}\right)+\frac{f(\kappa)}{\varepsilon}+o_{\alpha}(1)$,

where $0 \leq \hat{h}\left(x_{\varepsilon}, y_{\varepsilon}, \bar{x}, \bar{y}\right) \leq c$ for all $\varepsilon, 0<\varepsilon \leq \bar{\varepsilon}$, and for some non-negative function $f(\kappa)$ such that $f(\kappa) \rightarrow 0$ as $\kappa \rightarrow 0$. Note also that the points $\left\{\left(x_{\varepsilon}, y_{\varepsilon}, t_{\varepsilon}\right)\right\}_{\varepsilon<\bar{\varepsilon}}$ are independent of $\kappa$ and that the function $f(\kappa)$ is independent of $\varepsilon$. Assuming that (3.38) holds and combining the estimates in the last displays we see that

$$
\tilde{u}_{k}^{-}\left(x_{\varepsilon}, t_{\varepsilon}\right)-\bar{u}_{k}^{+}\left(y_{\varepsilon}, t_{\varepsilon}\right)+\frac{\theta}{t_{\varepsilon}^{2}}
$$




$$
\begin{aligned}
\leq & 2 \beta\left(t_{\varepsilon}-\bar{t}\right)+\frac{c}{\varepsilon}\left|x_{\varepsilon}-y_{\varepsilon}\right|^{2}+c \Lambda h\left(x_{\varepsilon}, y_{\varepsilon}, \bar{x}, \bar{y}\right)+c \Lambda \hat{h}\left(x_{\varepsilon}, y_{\varepsilon}, \bar{x}, \bar{y}\right) \\
& +\tilde{\psi}_{k}\left(x_{\varepsilon}, t_{\varepsilon}\right)-\tilde{\psi}_{k}\left(y_{\varepsilon}, t_{\varepsilon}\right)+\frac{f(\kappa)}{\varepsilon}+o_{\alpha}(1) .
\end{aligned}
$$

Now, letting first $\alpha \rightarrow 0$ and then $\kappa \rightarrow 0$ we get

$$
\begin{aligned}
& \tilde{u}_{k}^{-}\left(x_{\varepsilon}, t_{\varepsilon}\right)-\bar{u}_{k}^{+}\left(y_{\varepsilon}, t_{\varepsilon}\right)+\frac{\theta}{t_{\varepsilon}^{2}} \\
\leq & 2 \beta\left(t_{\varepsilon}-\bar{t}\right)+\frac{c}{\varepsilon}\left|x_{\varepsilon}-y_{\varepsilon}\right|^{2}+c \Lambda h\left(x_{\varepsilon}, y_{\varepsilon}, \bar{x}, \bar{y}\right)+c \Lambda \hat{h}\left(x_{\varepsilon}, y_{\varepsilon}, \bar{x}, \bar{y}\right) \\
& +\tilde{\psi}_{k}\left(x_{\varepsilon}, t_{\varepsilon}\right)-\tilde{\psi}_{k}\left(y_{\varepsilon}, t_{\varepsilon}\right) .
\end{aligned}
$$

Then, letting $\varepsilon \rightarrow 0$, using (3.21), 3.26) and the continuity of $\tilde{\psi}_{k}$, we can conclude that

$$
\tilde{u}_{k}^{-}(\bar{x}, \bar{t})-\bar{u}_{k}^{+}(\bar{x}, \bar{t})+\frac{\theta}{\bar{t}^{2}} \leq \tilde{c} \Lambda
$$

Finally, letting $\Lambda \rightarrow 0$ in the last display we can conclude that

$$
\tilde{u}_{k}^{-}(\bar{x}, \bar{t})-\bar{u}_{k}^{+}(\bar{x}, \bar{t})+\frac{\theta}{\bar{t}^{2}} \leq 0
$$

which contradicts 3.17)

To finish the proof we now only have to prove the estimate in (3.38). However, by following section 5 of $\mathrm{BI08}$, we see that we may replace $\mathcal{I}_{\kappa}\left(x_{\varepsilon}, t_{\varepsilon}, \varphi_{\varepsilon}^{\alpha}\left(\cdot, y_{\varepsilon}, t_{\varepsilon}\right), D_{x} \varphi_{\varepsilon}\right)$ by $\mathcal{I}_{\kappa}\left(x_{\varepsilon}, t_{\varepsilon}, \varphi_{\varepsilon}\left(\cdot, y_{\varepsilon}, t_{\varepsilon}\right), D_{x} \varphi_{\varepsilon}\right)+o_{\alpha}(1)$ and hence it suffices to prove (3.38) with $\varphi_{\varepsilon}^{\alpha}$ replaced by $\varphi_{\varepsilon}$. To do this we first see, using Taylor's formula, the definition of $\varphi_{\varepsilon},(1.10)$ and (1.11), that

$$
\begin{aligned}
& \mid\left(\mathcal{I}_{\kappa}\left(x_{\varepsilon}, t_{\varepsilon}, \varphi_{\varepsilon}\left(\cdot, y_{\varepsilon}, t_{\varepsilon}\right), D_{x} \varphi_{\varepsilon}\right)\right. \\
- & \left.\mathcal{I}_{\kappa}\left(y_{\varepsilon}, t_{\varepsilon},-\varphi_{\varepsilon}\left(x_{\varepsilon}, \cdot t_{\varepsilon}\right),-D_{y} \varphi_{\varepsilon}\right)\right) \mid \leq \frac{f(\kappa)}{\varepsilon}
\end{aligned}
$$

for all $\varepsilon, 0<\varepsilon \leq \bar{\varepsilon}$, and for some non-negative function $f(\kappa)$ as above. By the dominated convergence theorem we also have $f(\kappa) \rightarrow 0$ as $\kappa \rightarrow 0$ since

$$
\begin{aligned}
\lim _{\kappa \rightarrow 0} \int_{B(0, \kappa)}|z|^{2} \nu(d z) & =\lim _{\kappa \rightarrow 0} \int_{B(0,1)}|z|^{2} \chi_{\{|z| \leq \kappa\}} \nu(d z) \\
& =\int_{B(0,1)} \lim _{\kappa \rightarrow 0}\left(|z|^{2} \chi_{\{|z| \leq \kappa\}}\right) \nu(d z)=0 .
\end{aligned}
$$

Hence, only the term involving $\mathcal{I}^{\kappa}$ remains. To conduct estimates we first decompose

$$
\begin{aligned}
& -\left(\mathcal{I}^{\kappa}\left(x_{\varepsilon}, t_{\varepsilon}, \tilde{u}_{k}^{-}\left(\cdot, t_{\varepsilon}\right), D_{x} \varphi_{\varepsilon}\right)-\mathcal{I}^{\kappa}\left(y_{\varepsilon}, t_{\varepsilon}, \bar{u}_{k}^{+}\left(\cdot, t_{\varepsilon}\right),-D_{y} \varphi_{\varepsilon}\right)\right) \\
= & -\left(\mathcal{I}_{-}^{\kappa}\left(x_{\varepsilon}, t_{\varepsilon}, \tilde{u}_{k}^{-}\left(\cdot, t_{\varepsilon}\right), D_{x} \varphi_{\varepsilon}\right)-\mathcal{I}_{-}^{\kappa}\left(y_{\varepsilon}, t_{\varepsilon}, \bar{u}_{k}^{+}\left(\cdot, t_{\varepsilon}\right),-D_{y} \varphi_{\varepsilon}\right)\right) \\
& -\left(\mathcal{I}_{+}^{\kappa}\left(x_{\varepsilon}, t_{\varepsilon}, \tilde{u}_{k}^{-}\left(\cdot, t_{\varepsilon}\right), D_{x} \varphi_{\varepsilon}\right)-\mathcal{I}_{+}^{\kappa}\left(y_{\varepsilon}, t_{\varepsilon}, \bar{u}_{k}^{+}\left(\cdot, t_{\varepsilon}\right),-D_{y} \varphi_{\varepsilon}\right)\right)
\end{aligned}
$$

where $\mathcal{I}_{-}^{\kappa}$ indicates integration with respect to $z \in B(0,1) \backslash B(0, \kappa)$ and $\mathcal{I}_{+}^{\kappa}$ indicates integration with respect to $z \in \mathbb{R}^{l} \backslash B(0,1)$. Note that by construction, see (3.18), we can, working with index $k$, assume that the sequence $\left\{\left(x_{\varepsilon}, y_{\varepsilon}, t_{\varepsilon}\right)\right\}_{\varepsilon<\bar{\varepsilon}}$ is such that

$$
\begin{aligned}
\phi_{\varepsilon}^{k}\left(x_{\varepsilon}, y_{\varepsilon}, t_{\varepsilon}\right) & =\sup _{(x, y, t) \in \overline{B(0, R)} \times \overline{B(0, R)} \times(0, T)} \phi_{\varepsilon}^{k}(x, y, t), \text { where } \\
\phi_{\varepsilon}^{k}(x, y, t) & =\tilde{u}_{k}^{-}(x, t)-\bar{u}_{k}^{+}(y, t)-\frac{\theta}{t}-\varphi_{\varepsilon}(x, y, t) .
\end{aligned}
$$


To proceed we exploit that the maximizing property of the sequence $\left\{\left(x_{\varepsilon}, y_{\varepsilon}, t_{\varepsilon}\right)\right\}_{\varepsilon<\bar{\varepsilon}}$ in (3.41) implies, for $z \in \mathbb{R}^{l} \backslash B(0, \kappa)$, that

$$
\begin{aligned}
& \tilde{u}_{k}^{-}\left(x_{\varepsilon}+\eta\left(x_{\varepsilon}, t_{\varepsilon}, z\right), t_{\varepsilon}\right)-\tilde{u}_{k}^{-}\left(x_{\varepsilon}, t_{\varepsilon}\right)-\sum_{l} \eta_{l}\left(x_{\varepsilon}, t_{\varepsilon}, z\right) \partial_{x_{l}} \varphi_{\varepsilon}\left(x_{\varepsilon}, y_{\varepsilon}, t_{\varepsilon}\right) \\
& -\bar{u}_{k}^{+}\left(y_{\varepsilon}+\eta\left(y_{\varepsilon}, t_{\varepsilon}, z\right), t_{\varepsilon}\right)+\bar{u}_{k}^{+}\left(y_{\varepsilon}, t_{\varepsilon}\right)-\sum_{l} \eta_{l}\left(y_{\varepsilon}, t_{\varepsilon}, z\right) \partial_{y_{l}} \varphi_{\varepsilon}\left(x_{\varepsilon}, y_{\varepsilon}, t_{\varepsilon}\right) \\
\leq & \varphi_{\varepsilon}\left(x_{\varepsilon}+\eta\left(x_{\varepsilon}, t_{\varepsilon}, z\right), y_{\varepsilon}+\eta\left(y_{\varepsilon}, t_{\varepsilon}, z\right), t_{\varepsilon}\right)-\varphi_{\varepsilon}\left(x_{\varepsilon}, y_{\varepsilon}, t_{\varepsilon}\right) \\
& -\sum_{l} \eta_{l}\left(x_{\varepsilon}, t_{\varepsilon}, z\right) \partial_{x_{l}} \varphi_{\varepsilon}\left(x_{\varepsilon}, y_{\varepsilon}, t_{\varepsilon}\right)-\sum_{l} \eta_{l}\left(y_{\varepsilon}, t_{\varepsilon}, z\right) \partial_{y_{l}} \varphi_{\varepsilon}\left(x_{\varepsilon}, y_{\varepsilon}, t_{\varepsilon}\right) .
\end{aligned}
$$

To ease the notation in the calculations to follow we let $\eta_{x}^{\varepsilon}(z)=\eta\left(x_{\varepsilon}, t_{\varepsilon}, z\right), \eta_{y}^{\varepsilon}(z)=$ $\eta\left(y_{\varepsilon}, t_{\varepsilon}, z\right)$, and we let $\langle\cdot, \cdot\rangle$ denote that standard scalar product in $\mathbb{R}^{N}$. To proceed we first note here that the left hand side of (3.42) is precisely the integrand of

$$
\left(\mathcal{I}^{\kappa}\left(x_{\varepsilon}, t_{\varepsilon}, \tilde{u}_{k}^{-}\left(\cdot, t_{\varepsilon}\right), D_{x} \varphi_{\varepsilon}\right)-\mathcal{I}^{\kappa}\left(y_{\varepsilon}, t_{\varepsilon}, \bar{u}_{k}^{+}\left(\cdot, t_{\varepsilon}\right),-D_{y} \varphi_{\varepsilon}\right),\right.
$$

and hence, using (3.42), we see that we want develop appropriate estimates for the function

$$
\begin{aligned}
& \varphi_{\varepsilon}\left(x_{\varepsilon}+\eta_{x}^{\varepsilon}(z), y_{\varepsilon}+\eta_{y}^{\varepsilon}(z), t_{\varepsilon}\right)-\varphi_{\varepsilon}\left(x_{\varepsilon}, y_{\varepsilon}, t_{\varepsilon}\right) \\
& -\left\langle\left(\eta_{x}^{\varepsilon}(z), \eta_{y}^{\varepsilon}(z)\right),\left(D_{x} \varphi_{\varepsilon}\left(x_{\varepsilon}, y_{\varepsilon}, t_{\varepsilon}\right), D_{y} \varphi_{\varepsilon}\left(x_{\varepsilon}, y_{\varepsilon}, t_{\varepsilon}\right)\right)\right\rangle,
\end{aligned}
$$

where, as we recall,

$$
\varphi_{\varepsilon}(x, y, t)=\frac{1}{2 \varepsilon}|x-y|^{2}+\Lambda\left(|x-\bar{x}|^{2 \gamma+2}+|y-\bar{x}|^{2 \gamma+2}\right)+\beta(t-\bar{t})^{2} .
$$

A straight forward calculation shows that (3.44) can be simplified to

$$
\begin{aligned}
& \frac{1}{2 \varepsilon}\left|\eta_{x}^{\varepsilon}-\eta_{y}^{\varepsilon}\right|^{2} \\
& +\Lambda\left[A_{\varepsilon}-(2+2 \gamma)\left(\left\langle\eta_{x}^{\varepsilon},\left(x_{\varepsilon}-\bar{x}\right)\right\rangle\left|x_{\varepsilon}-\bar{x}\right|^{2 \gamma}+\left\langle\eta_{y}^{\varepsilon},\left(y_{\varepsilon}-\bar{x}\right)\right\rangle\left|y_{\varepsilon}-\bar{x}\right|^{2 \gamma}\right)\right]
\end{aligned}
$$

where

$$
A_{\varepsilon}:=\left[\left|x_{\varepsilon}+\eta_{x}^{\varepsilon}-\bar{x}\right|^{2+2 \gamma}-\left|x_{\varepsilon}-\bar{x}\right|^{2+2 \gamma}+\left|y_{\varepsilon}+\eta_{y}^{\varepsilon}-\bar{x}\right|^{2+2 \gamma}-\left|y_{\varepsilon}-\bar{x}\right|^{2+2 \gamma}\right] .
$$

We now first estimate the contribution to the expression in (3.43) coming from integration over $z \in B(0,1) \backslash B(0, \kappa)$, i.e., from the corresponding expression involving $\mathcal{I}_{-}^{\kappa}$. Given $z \in B(0,1) \backslash B(0, \kappa)$ and using (1.11), we first see that

$$
\frac{1}{2 \varepsilon}\left|\eta_{x}^{\varepsilon}-\eta_{y}^{\varepsilon}\right|^{2} \leq \frac{c}{\varepsilon}|z|^{2}\left|x_{\varepsilon}-y_{\varepsilon}\right|^{2}
$$

Concerning the second term of (3.45) we see, using the fundamental theorem of calculus and (1.11), that

$$
\begin{aligned}
& {\left[A_{\varepsilon}-(2+2 \gamma)\left(\left\langle\eta_{x}^{\varepsilon},\left(x_{\varepsilon}-\bar{x}\right)\right\rangle\left|x_{\varepsilon}-\bar{x}\right|^{2 \gamma}+\left\langle\eta_{y}^{\varepsilon},\left(y_{\varepsilon}-\bar{x}\right)\right\rangle\left|y_{\varepsilon}-\bar{x}\right|^{2 \gamma}\right)\right]} \\
& \leq c|z|^{2}\left(1+\left|x_{\varepsilon}-\bar{x}\right|^{2 \gamma+2}+\left|y_{\varepsilon}-\bar{x}\right|^{2 \gamma+2}\right)
\end{aligned}
$$

whenever $z \in B(0,1) \backslash B(0, \kappa)$. In particular, using the above estimates and (1.10) we can conclude that

$$
\begin{aligned}
& \left|\mathcal{I}_{-}^{\kappa}\left(x_{\varepsilon}, t_{\varepsilon}, \tilde{u}_{k}^{-}\left(\cdot, t_{\varepsilon}\right), D_{x} \varphi_{\varepsilon}\right)-\mathcal{I}_{-}^{\kappa}\left(y_{\varepsilon}, t_{\varepsilon}, \bar{u}_{k}^{+}\left(\cdot, t_{\varepsilon}\right),-D_{y} \varphi_{\varepsilon}\right)\right| \\
& \leq c\left(\frac{1}{\varepsilon}\left|x_{\varepsilon}-y_{\varepsilon}\right|^{2}+\Lambda\left(1+\left|x_{\varepsilon}-\bar{x}\right|^{2 \gamma+2}+\left|y_{\varepsilon}-\bar{x}\right|^{2 \gamma+2}\right)\right) .
\end{aligned}
$$

Finally, focusing on the contribution from the term $\mathcal{I}_{+}^{\kappa}$ we, through similar calculations as above now using (1.10) on $\mathbb{R}^{l} \backslash B(0,1)$, we find that (3.47) also holds with $\mathcal{I}_{-}^{\kappa}$ replaced by $\mathcal{I}_{+}^{\kappa}$ and hence the proof of (3.38), and Theorem 1.1, is complete. 


\section{Existence: Proof of Theorem 1.2 And Theorem 1.3}

4.1. Proof of Theorem 1.2. To prove Theorem 1.2 we use Perron's method. To construct a viscosity solution to problem (1.1) using Perron's method we define $u=\left(u_{1}, \ldots, u_{d}\right)$ through

$$
u_{i}(x, t):=\inf \left\{u_{i}^{+}(x, t):\left(u_{1}^{+}, \ldots, u_{d}^{+}\right) \text {is a supersolution of problem (1.1) }\right\} \text {. }
$$

Note that $u=\left(u_{1}, \ldots, u_{d}\right)$ is well-defined based on the assumption of the existence of a barrier from above. Let $u^{*}=\left(u_{1}^{*}, \ldots, u_{d}^{*}\right)$ and $u_{*}=\left(u_{*, 1}, \ldots, u_{d, *}\right)$ denote, respectively, the upper and lower semi-continuous envelopes of $u$, i.e.,

$$
\begin{aligned}
u_{i}^{*}(x, t) & =\limsup _{r \rightarrow 0}\left\{u_{i}(y, s):(y, s) \in(B(x, r) \times(t-r, t+r)) \cap\left(\mathbb{R}^{N} \times(0, T]\right)\right\}, \\
u_{i, *}(x, t) & =-\left(-u_{i}(x, t)\right)^{*},
\end{aligned}
$$

for $i \in\{1, \ldots, d\}$. Then, by definition and by Theorem 1.1 we have

$$
u_{i}^{-} \leq u_{i}^{*}, \quad u_{i, *} \leq u_{i}^{+} \quad \text { and } \quad u_{i, *} \leq u_{i}^{*},
$$

for all $i \in\{1, \ldots, d\}$, and where $u^{-}=\left(u_{1}^{-}, \ldots, u_{d}^{-}\right)$and $u^{+}=\left(u_{1}^{+}, \ldots, u_{d}^{+}\right)$are sub- and supersolutions to (1.1), respectively. The essence of Perron's method is now to prove that $u^{*}$ and $u_{*}$ are, respectively, a subsolution and a supersolution to problem (1.1). It then follows by the comparison principle that $u^{*} \leq u_{*}$ and hence $u=u_{*}=u^{*}$ is a viscosity solution to the system in (1.1). We first prove that $u^{*}$ assumes the correct terminal data. To do this, fix a component $i \in\{1, \ldots, d\}$ and a point $y \in \mathbb{R}^{N}$. By assumption, there exists a barrier from above $u^{+}=u^{+, i, y}$ to the system in (1.1) in the sense of Definition 1. In particular, by definition $u_{i}(x, t) \leq u_{i}^{+, i, y, \varepsilon}(x, t)$ whenever $(x, t) \in \mathbb{R}^{N} \times[0, T]$ and for all $\varepsilon>0$. Furthermore, since $u^{+, i, y, \varepsilon}$ is continuous we have that $u_{i}^{*}(x, t) \leq\left(u_{i}^{+, i, y, \varepsilon}\right)_{i}^{*}(x, t)=u_{i}^{+, i, y, \varepsilon}(x, t)$ for every $\varepsilon>0$ and $(x, t) \in \mathbb{R}^{N} \times[0, T]$. In particular, for component $i$ and the point $y$ we have that

$$
u_{i}^{*}(y, T) \leq \lim _{\varepsilon \rightarrow 0} u_{i}^{+, i, y, \varepsilon}(y, T)=g_{i}(y) .
$$

Since $i$ and $y$ are arbitrary in this argument we conclude that $u^{*}$ assumes the correct terminal data. That $u_{*}$ also assumes the correct terminal data is proved similarly using the assumption of barriers from below. Hence it only remains to prove that the remaining conditions for the property of being sub- and supersolutions to the system hold for $u^{*}$ and $u_{*}$, respectively. However, after noticing that our switching costs are assumed to be continuous, see (1.12), this follows in the same way, for the subsolution, as outlined on p. 70 in BJK10, and for the supersolution, as outlined on p.71-72 in BJK10. We omit further details and conclude that the proof of Theorem 1.2 is complete.

4.2. Proof of Theorem 1.3. In the light of Theorem 1.2 we see that to prove Theorem 1.3 we only need to construct barriers from above and below, in the sense of Definition 1, for each $i \in\{1, \ldots, d\}$ and $y \in \mathbb{R}^{N}$. To construct an appropriate barrier from above, for fixed $i \in\{1, \ldots, d\}$ and $y \in \mathbb{R}^{N}$, we let, for all $j \in\{1, \ldots, d\}$,

$$
\begin{aligned}
u_{j}^{+, i, y, \varepsilon}(x, t)= & g(y)+\frac{K}{\varepsilon^{2}}(T-t) \\
& +L\left(e^{\lambda(T-t)}+1\right)\left(|x-y|^{2}+\varepsilon\right)^{\frac{1}{2}}+c_{i, j}(x, t),
\end{aligned}
$$

where $K$ and $\lambda$ are non-negative degrees of freedom and $L$ is the Lipschitz-constant of $g(x)$. We first note that (1.14), and the assumption that $g_{i}=g$ for all $i \in$ $\{1, \ldots, d\}$, implies that $c_{i, j}(x, T) \geq 0$ for all $i, j \in\{1, \ldots, d\}$. Hence

$$
u^{+, i, y, \varepsilon}(x, T)=g(y)+2 L\left(|x-y|^{2}+\varepsilon\right)^{\frac{1}{2}}+c_{i, j}(x, T) \geq g(x),
$$


whenever $x \in \mathbb{R}^{N}$ and, in particular,

$$
u_{i}^{+, i, y, \varepsilon}(y, T)=g(y)+2 L \varepsilon^{\frac{1}{2}}+c_{i, i}(x, T) .
$$

Hence, using (1.13) $(i)$ we see that $u_{i}^{+, i, y, \varepsilon}(y, T)=g(y)+2 L \varepsilon^{\frac{1}{2}}$ and

$$
\lim _{\varepsilon \rightarrow 0} u_{i}^{+, i, y, \varepsilon}(y, T)=g(y) .
$$

We next verify that $u^{+, i, y, \varepsilon}$ is actually a supersolution to 1.1). To do this we start by observing that assumption (1.15) implies that $u^{+, i, y, \varepsilon}$ is above the obstacle. In particular,

$$
\begin{aligned}
& u_{j}^{+, i, y, \varepsilon}-\max _{k \neq j}\left(-c_{j, k}(x, t)+u_{k}^{+, i, y, \varepsilon}\right) \\
= & -\max _{k \neq j}\left(-c_{i, j}(x, t)-c_{j, k}(x, t)+c_{i, k}(x, t)\right) \geq 0,
\end{aligned}
$$

where the last inequality is a consequence of (1.15). Hence, to complete the proof we only have to verify that

$$
\begin{aligned}
-\mathcal{H} u_{j}^{+, i, y, \varepsilon}(x, t)-\psi_{j}(x, t)= & -\mathcal{L} u_{j}^{+, i, y, \varepsilon}(x, t) \\
& -\mathcal{I}\left(x, t, u_{j}^{+, i, y, \varepsilon}\right)-\psi_{j}(x, t) \geq 0 .
\end{aligned}
$$

Using assumptions (1.8), (1.9), and (1.16) we deduce by standard calculations that

$$
\begin{aligned}
& \quad\left|\sum_{k, l=1}^{N} a_{k, l}(x, t) \partial_{x_{k} x_{l}} u^{+, i, y, \varepsilon}+\sum_{k=1}^{N} a_{k}(x, t) \partial_{x_{k}} u^{+, i, y, \varepsilon}\right| \\
& \leq c\left(e^{\Lambda(T-t)}+1\right)\left(1+\frac{1}{\varepsilon^{2}}+|x|\right),
\end{aligned}
$$

where the constant $c$ is independent from $K$. Concerning the non-local term $\mathcal{I}\left(x, t, u_{j}^{+, i, y, \varepsilon}\right)$, using Taylor's formula and assumption (1.11), we deduce, for $|z| \leq$ 1 ,

$$
\begin{aligned}
& \left|u_{j}^{+, i, y, \varepsilon}(x+\eta(x, t, z), t)-u_{j}^{+, i, y, \varepsilon}(x, t)-\sum_{l} \eta_{l}(x, t, z) \partial_{x_{l}} u_{j}^{+, i, y, \varepsilon}(x, t)\right| \\
& \leq c|z|^{2} \sup _{x^{\prime} \in \mathbb{R}^{N}}\left\|\nabla_{x}^{2} u_{j}^{+, i, y, \varepsilon}\left(x^{\prime}, t\right)\right\|,
\end{aligned}
$$

and, for $|z|>1$,

$$
\begin{aligned}
& \left|u_{j}^{+, i, y, \varepsilon}(x+\eta(x, t, z), t)-u_{j}^{+, i, y, \varepsilon}(x, t)-\sum_{l} \eta_{l}(x, t, z) \partial_{x_{l}} u_{j}^{+, i, y, \varepsilon}(x, t)\right| \\
& \leq c \sup _{x^{\prime} \in \mathbb{R}^{N}}\left\|\nabla_{x}^{2} u_{j}^{+, i, y, \varepsilon}\left(x^{\prime}, t\right)\right\| .
\end{aligned}
$$

It is clear from the construction of $u_{j}^{+, i, y, \varepsilon}$, see (4.2), and (1.16) that

$$
\sup _{x^{\prime} \in \mathbb{R}^{N} \backslash B(y, 1)}\left\|\nabla_{x}^{2} u_{j}^{+, i, y, \varepsilon}\left(x^{\prime}, t\right)\right\| \leq c
$$

and hence we only need to consider values of $x$ such that $|x-y|<1$. Straightforward calculations using (1.11) show that

$$
\sup _{x^{\prime} \in B(y, 1)}\left\|\nabla_{x}^{2} u_{j}^{+, i, y, \varepsilon}\left(x^{\prime}, t\right)\right\| \leq c\left(e^{\Lambda(T-t)}+1\right)\left(\frac{1}{\varepsilon^{\frac{3}{2}}}+\frac{1}{\varepsilon^{\frac{1}{2}}}\right) \leq \frac{c}{\varepsilon^{2}}\left(e^{\Lambda(T-t)}+1\right),
$$

where, again, the constant $c$ is independent from $K$. Hence, taking assumption (1.10) into account we see that

$$
\left|-\mathcal{I}\left(x, t, u_{j}^{+, i, y, \varepsilon}\right)\right| \leq \frac{c}{\varepsilon^{2}}\left(e^{\Lambda(T-t)}+1\right) .
$$


Next, note that

$$
\partial_{t} u_{j}^{+, i, y, \varepsilon}=-\frac{K}{\varepsilon^{2}}-\Lambda e^{\Lambda(T-t)}\left(|x-y|^{2}+\varepsilon\right)^{\frac{1}{2}}+\partial_{t} c_{i, j} .
$$

Putting the above estimates together and using (1.16) we find that

$$
\begin{aligned}
\mathcal{H} u_{j}^{+, i, y, \varepsilon}(x, t) & -\psi_{j}(x, t) \\
& \geq \frac{K}{\varepsilon^{2}}+\Lambda e^{\Lambda(T-t)}\left(|x-y|^{2}+\varepsilon\right)^{\frac{1}{2}}-c e^{\Lambda(T-t)}\left(1+\frac{1}{\varepsilon^{2}}+|x|\right) \geq 0
\end{aligned}
$$

for $K$ and $\Lambda$ large enough. Hence $u^{+, i, y, \varepsilon}$ is a supersolution to (1.1).

To construct an appropriate barrier from below, for fixed $i \in\{1, \ldots, d\}$ and $y \in \mathbb{R}^{N}$, we let, for all $j \in\{1, \ldots, d\}$,

$$
\begin{aligned}
u_{j}^{-, i, y, \varepsilon}(x, t)= & g(y)-\frac{K}{\varepsilon^{2}}(T-t) \\
& -L\left(e^{\lambda(T-t)}+1\right)\left(|x-y|^{2}+\varepsilon\right)^{\frac{1}{2}}-c_{i, j}(x, t),
\end{aligned}
$$

where again $K$ and $\lambda$ are non-negative degrees of freedom and $L$ is the Lipschitzconstant of $g(x)$. The above argument can then be repeated to conclude that $u^{-, i, y, \varepsilon}$ is a subsolution to (1.1) and hence the proof of Theorem 1.3 is complete. We omit further details.

Remark 4.1. Note that a local version of Theorem 1.3 is proved as Theorem 2.4 in LNO12. In the proof of Theorem 2.4 in LNO12 the barriers in (4.2) and (4.6) are also used. However, there are two typo errors in the statements of these barriers in LNO12. Indeed, the factors $e^{-\lambda t}$ and $K$ stated in the corresponding construction in LNO12 should be corrected and replaced by $e^{\lambda(T-t)}$ and $\frac{K}{\varepsilon^{2}}$ respectively, as above. The subsequent calculation/argument in [LNO12 should also be modestly adjusted accordingly.

\section{REFERENCES}

[A07] A.-L. Amadori Obstacle problem for nonlinear integro-differential equations arising in option pricing Ricerche di Matematica, 56(2007), 1-17.

[AF12] B. El-Asri, I. Fakhouri, Optimal multi-modes switching with the switching cost not necessarily positive, arXiv:1204.1683v1, 2012.

[AH09] B. El-Asri and S. Hamadene, The finite horizon optimal multi-modes switching problem: The viscosity solution approach Applied Mathematics \& Optimization, 60(2009), 213-235.

[BI08] G., Barles, C. Imbert, Second-order elliptic integro-differential equations: viscosity solutionstheory revisited, Annales de línstitut Henri Poincaré, 25 (2008), 567-585.

[BJK10] I. H. Biswas, E. R. Jakobsen, K. H. Karlsen, Viscosity solutions for a system of integroPDEs and connections to optimal switching and control of jump-diffusion processes, Applied Mathematics \& Optimization, 62 (2010), 47-80.

[DH09] B. Djehiche, S. Hamadene, On A Finite Horizon Starting And Stopping Problem With Risk Of Abandonment, International Journal of Theoretical and Applied Finance, 12 (2009), 523-543.

[DHP10] B. Djehiche, S. Hamadene, A. Popier, A Finite Horizon Optimal Multiple Switching Problem, SIAM Journal on Control and Optimization, 48 (2010), 2751-2770

[HM12] S. Hamadéne, M.A. Morlais Viscosity Solutions of Systems of PDEs with Interconnected Obstacles and Multi-Modes Switching Problem, arXiv:1104.2689v2.

[HT07] Y. Hu, S. Tang Multi-dimensional BSDE with Oblique Reflection and Optimal Switching, Probability Theory and Related Fields, 147 (2010), 89-121.

[HZ10] S. Hamadéne, J. Zhang Switching Problem and Related System of Reflected Backward SDEs, Stochastic Processes and their Applications, 120 (2010), 403-426.

[IK91] H. Ishii, S. Koike, Viscosity Solutions of a System of Nonlinear Second-Order Elliptic PDEs Arising in Switching Games, Funkcialaj Ekvacioj, 34 (1991), 143-155.

[JK05] E. R. Jakobsen, K. H. Karlsen, Continuous Dependence Estimates for Viscosity Solutions of Integro-PDEs, Journal of Differential Equations, 212 (2005), 278-318. 
[JK06] E. R. Jakobsen, K. H. Karlsen, A Maximum Principle for Semicontinuous Functions Applicable to Integro-Partial Differential Equations, Nonlinear differential equations and applications, 13 (2006), 137-165.

[LNO12] N. Lundström, K. Nyström, M. Olofsson, Systems of variational inequalities in the context of Optimal Switching Problems and Operators of Kolmogorov Type, to appear in Annali di Mathematica Pura ed Applicata.

[P97] H. Pham, Optimal stopping, free boundary, and American option pricing in a jumpdiffusion model, Applied Mathematics \& Optimization, 35 (1997), 145-164.

Niklas L. P. Lundström, Department of Mathematics, Uppsala University, S-75106 UPPSALA, SWEDEN

E-mail address: niklas.lundstrom@math.umu.se

Kaj Nyström, Department of Mathematics, Uppsala University, S-751 06 Uppsala, SWEDEN

E-mail address: kaj.nystrom@math.uu.se

Marcus Olofsson, Department of Mathematics, Uppsala University, S-751 06 UppSALA, SWEDEN

E-mail address: marcus.olofsson@math.uu.se 